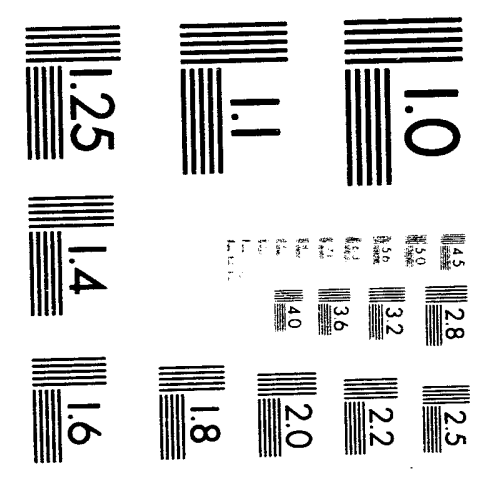



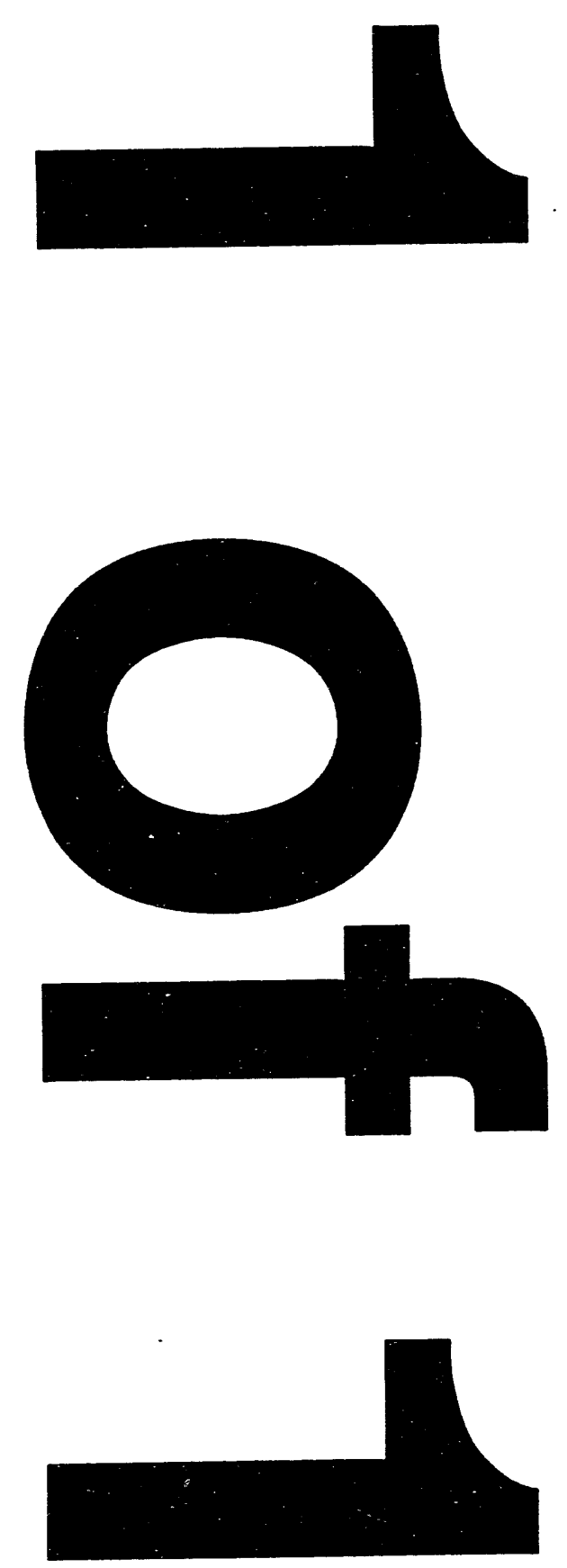


\title{
THREE-DIMENSIONAL EFFECTS FOR RADIO FREQUENCY ANTENNA MODELING
}

\author{
M. D. Carter \\ D. B. Batchelor \\ D. C. Stallings
}

DATE PUBLISHED - September 1993

Prepared for the

Office of Fusion Energy

Budget Activity AT 0520210

Prepared by

OAK RIDGE NATIONAL LABORATORY

Oak Ridge, Tennessee 37831-6285

managed by

MARTIN MARIETTA ENERGY SYSTEMS, INC.

for the

U.S. DEPARTMENT OF ENERGY

under contract DE-AC05-84OR21400

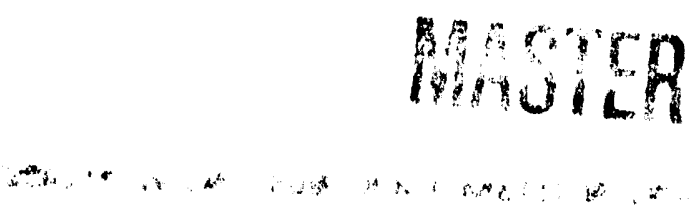




\section{CONTENTS}

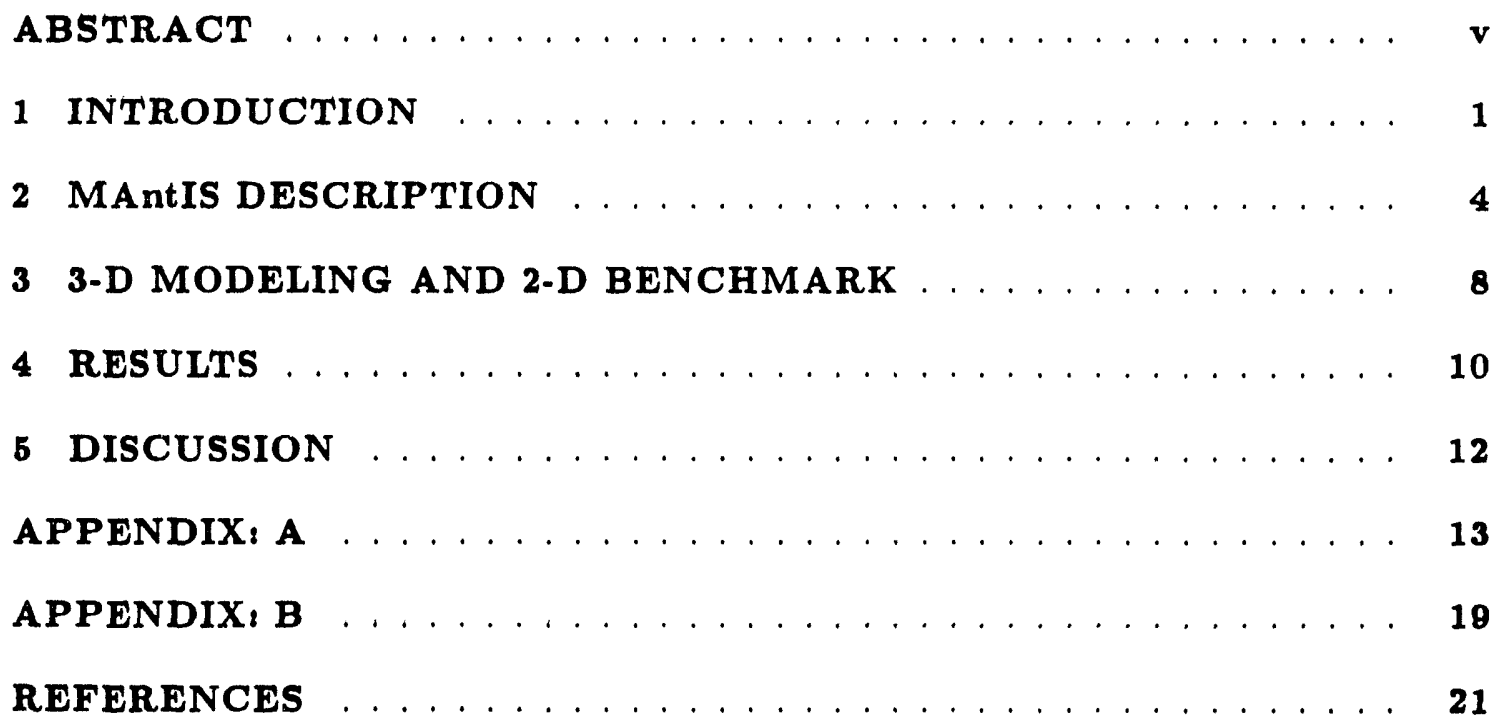




\begin{abstract}
Electromagnetic field calculations for radio frequency (rf) antennas in two dimensions (2-D) neglect finite antenna length effects as well as the feeders leading to the main current strap. Comparisons with experiments indicate that these 2-D calculations can overestimate the loading of the antenna and fail to give the correct reactive behavior. The 2-D calculations also predict that the return currents in the sidewalls of the antenna structure depend strongly on plasma parameters, but this prediction is also suspect because of experimental evidence.

To study the validity of the 2-D approximation, the Multiple Antenna Implementation System (MAntIS) has been used to perform 3-D modeling of the power spectrum, plasma loading, and inductance for a relevant loop antenna design. Effects on antenna performance caused by feeders to the main current strap, conducting sidewalls, and finite phase velocity are considered. The plasma impedance matrix for the loading calculation is generated by use of the ORION-1D code. The 3-D model is benchmarked with the 2-D model in the 2-D limit.

For finite-length antennas, inductance calculations are found to be in much more reasonable agreement with experiments for 3-D modeling than for the 2-D estimates. The modeling shows that the feeders affect the launched power spectrum in an indirect way by forcing the driven of current to return in the antenna sidewalls rather than in the plasma as in the 2-D model. Thus, the feeders have much more influence than the plasma on the currents that return in the sidewall. It has also been found that poloidal dependencies in the plasma impedance matrix can reduce the loading from that predicted in the 2-D model. For some plasma parameters, the combined 3-D effects can lead to a reduction in the predicted loading by as much as a factor of 2 from that given by the 2-D model.
\end{abstract}




\section{INTRODUCTION}

Waves with frequencies in the ion cyclotron (or gyro) range of frequencies (ICRF) have been successfully used to heat plasmas and modify the operation of experimental fusion devices. These radio frequency ( $\mathrm{rf}$ ) systems are attractive for many applications in fusion reactors because they are reliable, effective, and relatively inexpensive. Applications for reactors include the driving of steady-state currents, the stabilization of sawteeth, and the auxiliary heating of plasma to ignition.

The coupling between rf antennas and plasma is often modeled in two dimensions with variation in only the radial and toroidal directions [1]. This two-dimensional (2-D) approximation eliminates any poloidal variation in the system and models the antenna as a loop of current running around the entire poloidal extent of the plasma. Thus, the only poloidal mode number, $m$, considered in the 2-D model is $m=0$.

Concerns about the accuracy of this 2-D approximation arise from two physical considerations. First, the cutoff density for the fast wave increases with increasing poloidal mode number. Thus, the 2-D model can significantly overestimate the antenna loading for some plasma parameters because an antenna with finite poloidal length can generate a significant amount of power in modes with $m \neq 0$. A three-dimensional (3-D) model is necessary to consider modes with $m=0$. Second, the 2-D model permits the plasma to respond uniformly over its entire poloidal extent. This freedom allows the plasma to carry an image current that appears to "rob" current from the driven strap when viewed from a distance that is greater than the separation between the strap and the plasma. As the plasma "robs" current from the main strap in the 2-D model, nearby metallic structures carry a reduced image current in response to the combined plasma and driven strap currents. In three dimensions, feeders for the driven strap force currents to be returned in the antenna structure. Thus, the return currents in the 3-D model are much less sensitive to the current carried by the plasma than in the 2-D model. This inaccuracy in the 2-D estimate for the return currents can be important when calculating the toroidal power spectrum launched by phased array antennas for current drive applications.

The intention in this paper is to isolate the most important 3-D effects with a simplified model. The dominant 3-D effects are illustrated by considering a schematic of a typical antenna in relation to a tokamak plasma as shown in Fig. 1. Although the antenna is fully three-dimensional, a full 3-D solution is not necessary over the entire domain of the tokamak because the plasma does not vary in the toroidal direction (along the direction of the magnetic axis). This fact immediately permits the use of periodic Fourier analysis in the toroidal direction for solutions in the plasma region. The plasma is also weakly varying in the poloidal direction, and neglecting this poloidal variation permits solutions in terms of a set of uncoupled ordinary differential equations in the radial direction. Thus, in the plasma region, a single equation can be solved in the radial direction for each toroidal and poloidal mode.

A poloidal cross-section of an antenna that might be used in present-day tokamak designs is shown in Fig. 2. The main feature shown in Fig. 2 is the driven current element. This element consists of the current strap and the feeders that connect the current strap to capacitors through a feedthrough in the conducting backwall. The current element in this design is driven by an rf transmitter such that the current amplitude is at a maximum near the center of the current strap. Also shown in Fig. 2 is the Faraday shield housing. 
The housing typically consists of a solid conducting box including sidewalls for the antenna (see Fig. 1) and septa that separate current elements in multiple element designs. This conducting box is electrically connected to the the conducting backwall. Note that in some designs, the conducting box does not entirely enclose the main current strap but does cover at least most of the feeders.

This paper considers 3-D effects arising from two features that are illustrated in Fig. 2. One feature is the plasma response to a current strap having finite poloidal extent; modes with poloidal variation are excited by a finite-length antenna and have reduced wave number in the radial direction. This feature is also of concern because the plasma cannot respond to the antenna structure with simple currents flowing the entire poloidal distance around the plasma. A second feature requiring 3-D analysis is the inductive coupling that occurs between the current element and the conducting box (Faraday screen housing). This coupling is three-dimensional because the feeders effectively provide a short to the conducting backwall. These shorts produce radial image currents in the sides, bottom, and top of the conducting box that affect the launched power spectrum. Neither of these effects is included in a $2-D$ model.

Other features to be considered in a general 3-D model are shown in Fig. 2 but are not explicitly considered in this paper. These features include the Faraday shield face tubes and the protective bumper tile. The bumper tiles protect the Faraday shield tubes from plasma in the scrapeoff layer and provide a sharp boundary for the plasma-vacuum interface. The Faraday shield tubes are difficult to model explicitly because they represent a very strong modulation in the poloidal direction; however, this modulation is very localized in the radial direction. Magnetic flux passes easily through a well-designed Faraday shield, and its primary function is to isolate electrostatic fields (mostly in the radial direction) from the plasms. Thus, explicit modeling of the Faraday shield tubes is necessary only when details for the fields near the tubes are needed. The electrostatic fields between the strap and the shield tubes can be estimated by allowing for the variacion of the current along the strap. Transmission-line analysis [2] is frequently used to described this variation using a finite phase velocity, $v_{\phi}$, where $v_{\phi}=\lambda \omega / 2 \pi$ and $\lambda$ is the effective wavelength describing the current variation along the strap. Capacitive effects from the Faraday shield can cause $v_{\phi}$ to be significantly less than the speed of light in a vacuum.

To analyze the dominant physical effects arising in $3-\mathrm{D}$, we model the layer between the plasma and the conducting vacuum vessel shown in Figs. 1 and 2 . This layer contains the 3-D antenna structure, and we treat this layer as a perindic slab in vacuum. We consider the radial direction with the Cartesian coordinate, $x$, as shown in Fig. 3. The Multiple Antenna Implementation System (MAntIS) code [3] is used to calculate the electromagnetic fields in this vacuum layer for multiple current elements over a ground plane (see Fig. 4). The elements can have nearly arbitrary orientation, and multiple elements are treated by the method of superposition. These elements include currents in the poloidal and toroidal directions at a fixed $x$ location, and also include feeders that carry current in the $x$ direction to the ground plane. These feeders are chosen such that the current is continuous at the corners of the current element.

The MAntIS code treats the plasma surface at $x=0$ in Fig. 3 as a surface impedance. The plasma surface impedance is calculated in the same manner as that used by Brambilla [4] using a slab plasma model (radial variation of plasma parameters with uncoupled poloidal and toroidal modes). The use of a slab plasma model is justified for the case of good 
wave absorption in the plasma region, and the impedance match is described in detail in Appendices A and B. The surface impedance is calculated once for a set of plasma parameters using the ORION-1D code [5] and need not be recalculated for any change in the current distribution in the vacuum layer. The Fourier analysis of the current elements used by the MAntIS code is described in Sect. 2. The electric field solutions for these elements are described in Appendix A.

The modeling is performed by placing current elements near the locations that are known to carry significant rf currents. The currents carried by the current elements are then prescribed in the model with no attempt at a fully self-consistent solution [6]. However, the amplitudes and variation of the currents along the strep are adjusted to approximately satisfy the appropriate boundary conditions for the antenua. The MAntIS code provides rapid solutions for a known distribution of currents in the vacuum layer by using analytic solutions to Maxwell's equations for eacil mode in Fourier space. Fields in real space are obtained from the Fourier solutions using fast Fourier techniques. Diagnostics pertaining to the boundary conditions and visualization of the fields in real space are performed with postprocessing programs.

The remainder of the paper is organized as follows. Section 2 contains a brief description of the MAntIS code explaining the basic current elements used to model rf systems. Section 3 describes the current elements used in the 3-D modeling of two side-by-side rf antenna structures including a septum. The model is chosen to be representative of antennas selected for experiments in TPX. Section 3 also describes how the 3-D model behaves in the 2-D limit and gives results from benchmarking the 3-D model with an existing 2-D antenna code RAivT [1]. Section 4 describes results obtained from the 3-D model and compares the results with predictions by the $2-\mathrm{D}$ model. These results show that $3-\mathrm{D}$ effects can lead to significant differences in the launched power spectrum from the spectrum predicted by the 2-D model. The primary source of these differences is traced to return currents that have part of their path along the third dimension. A discussion and a summary of the results are presented in Sect. 5. Appendix A gives a detailed description of the analytic solutions for the basic current elements used in the MAntIS code. Appendix B describes the generation of the plasma impedance matrix. 


\section{MAntIS DESCRIPTION}

Maxwell's equations for fields of the form $\vec{E} \exp (-i \omega t)$ and known current distributions are given by

$$
\nabla^{2} \vec{E}+\frac{\omega^{2}}{c^{2}} \vec{E}-\nabla(\nabla \cdot \vec{E})=-i \omega \mu_{0} \vec{J}
$$

where $\vec{E}$ and $\vec{J}$ are complex quantities referring to the electric field and the current, respectively. Following Figs. 1 and 2 and as discussed in Sect. 1, MAntIS solves Eq. (1) near the antenna, assuming that the region can be modeled as a periodic slab.

Using a Cartesian coordinate system with $y$ in the poloidal direction and $z$ in the toroidal direction, we expand the components of $\vec{E}$ and $\vec{J}$ using a complete periodic basis over the range $-\pi a \leq y \leq \pi a$ and $-\pi R \leq z \leq \pi R$, e.g.,

$$
\vec{J}(x, y, z)=\sum_{m, n} \vec{J}^{m, n}(x) \exp \{i[(m y / a)+(n z / R)]\},
$$

where the coefficients are given by

$$
\vec{J}^{m, n}(x)=\frac{1}{4 \pi^{2} a R} \int_{-\pi a}^{\pi a} \int_{-\pi R}^{\pi R} \vec{J}(x, y, z) \exp \{-i[(m y / a)+(n z / R)]\} d z d y
$$

and where $m$ and $n$ are integers ranging from $-\infty$ to $\infty$. This expansion represents a slab model for a torus with minor radius $a$ and major radius $R$. (The explicit $x$ dependence notation will be dropped in most instances but is retained for emphasis in some equations.)

Charge conservation and Coulomb's law, $\nabla \cdot \vec{J}=i \omega \rho=i \omega \nabla \cdot \vec{E} /\left(\mu_{0} c^{2}\right)$, can be used in Eq. (1) to obtain second-order ordinary differential equations for the $y$ and $z$ componer.ts of $\vec{E}$,

$$
\begin{aligned}
& \frac{d^{2} E_{y}^{m, n}}{d x^{2}}+k_{\perp}^{2 m, n} E_{y}^{m, n}=-i \omega \mu_{0}\left[J_{y}^{m, n}+\frac{i c^{2}}{\omega^{2}} \frac{m}{a}(\nabla \cdot \vec{J})^{m, n}\right] \\
& \frac{d^{2} E_{z}^{m, n}}{d x^{2}}+k_{\perp}^{2 m, n} E_{z}^{m, n}=-i \omega \mu_{0}\left[J_{z}^{m, n}+\frac{i c^{2}}{\omega^{2}} \frac{n}{R}(\nabla \cdot \vec{J})^{m, n}\right],
\end{aligned}
$$

where $k_{\perp}^{2 m, n}=\omega^{2} / c^{2}-m^{2} / a^{2}-n^{2} / R^{2}$, and the notation $(\nabla \cdot \vec{J})^{m, n}$ refers to the Fourier components of the time derivative of the charge density, $i \omega \rho^{m, n}(x)$. Solutions to Eqs. $(4 a)$ and (4b) can be found if two boundary conditions for $E_{y}^{m, n}$ and $E_{z}^{m, n}$ are known, provided that $\vec{J}$ is specified. The $E_{\boldsymbol{r}}^{m, n}$ component can be determined in terms of the solutions to Eqs. (4a) and (4b) by Fourier analysis of the $x$ component of Eq. (1), giving

$$
k_{\perp}^{2 m, n} E_{x}^{m, n}=-i \omega \mu_{0} J_{x}^{m, n}+\frac{i m}{a} \frac{d E_{y}^{m, n}}{d x}+\frac{i n}{R} \frac{d E_{z}^{m, n}}{d x} .
$$

One of the boundary conditions needed to solve Eqs. (4a) and (4b) is an impedance relationship between $\dot{x} \times \vec{E}$ and $\dot{x} \times \vec{B}$ at the plasma surface, $x=0$ (see Fig. 3 ), where $\vec{B}$ is the rf magnetic field. This plasma surface impedance effectively determines the spectrum for power flow through the plasma surface for any prescribed $\vec{B}$ by Poynting's theorem. The surface impedance is calculated using a modified version of the ORION-1D code [5]. 
The ORION-1D code solves Eq. (1) in a periodic slab using the warm plasma conductivity tensor to calculate the plasma currents in the plasma region $(x<0)$. The impedance matrix is generated by specifying the value of $\hat{x} \times \vec{B}^{m, n}$ for a single pair of wavenumber indices, $m$, and $n$ at $x=0$ and using this value as the source term for the ORION-1D code. The ORION-1D code calculates the resulting values for $E_{y}^{m, n}$ and $E_{z}^{m, n}$ throughout the plasma region using finite-difference techniques and an artificial absorber to prevent reflections from the conducting wall on the far side of the plasma slab. The values for the impedance matrix are determined from the ORION-1D solution by taking the ratio of $E_{y}^{m, n}$ or $E_{z}^{m, n}$ at $x=0$ to the source magnetic field component. The details of this calculation are given in Appendix $B$. The second boundary condition required to solve Eqs. (4a) and (4b) is obtained by specifying the value of $E_{y}$ and $E_{z}$ at $x=d+a_{w}$ (see Fig. 3). Typically the choice for this boundary condition is $E_{y}\left(x=d+a_{w}\right)=0$ and $E_{z}\left(x=d+a_{w}\right)=0$, representing a conducting ground plane at $x=d+a_{w}$.

A superposition of basic current elements is used to model complicated antenna structures in MAntIS. These basic elements, illustrated in Fig. 4, describe the flow of current on the surface of rectangular parallelepipeds. The elements are represented using the gradient of a geometry function, $f(x, y, z)$, that has a value of 1 inside the parallelepiped and of 0 outside. To prescribe $\vec{J}$ using $f$, we introduce a Cartesian coordinate system, $\left(x^{\prime}, y^{\prime}, z^{\prime}\right)$, associated with each element, as shown in Fig. 5. This coordinate system has its origin at the center of the element and is translated relative to the coordinates for the plasma by the values $y_{c}$ and $z_{c}$. The element can be rotated around the $x$-axis by an angle, $\theta$, measured from the $z$-axis, so that currents with nearly arbitrary orientation relative to the plasma can be considered as shown in Fig. 5. The transformation from the plasma coordinate system to the element coordinate system is $x=x^{\prime}, y=y_{c}+y^{\prime} \cos (\theta)+z^{\prime} \sin (\theta)$, and $z=z_{c}-y^{\prime} \sin (\theta)+z^{\prime} \cos (\theta)$. Heaviside step functions, $\Theta$, are used to describe $f$ :

$$
\begin{array}{r}
f\left(x^{\prime}, y^{\prime}, z^{\prime}\right)=\Theta\left(x^{\prime}-d\right)\left[\Theta\left(y^{\prime}+\frac{\alpha}{2}\right)-\Theta\left(y^{\prime}-\frac{\alpha}{2}\right)\right] \\
\times\left[\Theta\left(z^{\prime}+\frac{\beta}{2}\right)-\Theta\left(z^{\prime}-\frac{\beta}{2}\right)\right] .
\end{array}
$$

The direction of the current flow for each element is described by $\hat{f} \times \hat{y}^{\prime}$, where $\hat{f} \equiv \nabla f /|\nabla f|$ and $\hat{y}^{\prime}$ is the unit vector in the $y^{\prime}$ direction, as shown in Fig. 3. Note that the feeders are included in the basic element and are arbitrarily chosen to be at $z^{\prime}= \pm \beta / 2$. This representation simplifies the model by constraining the currents to flow in the $\boldsymbol{z}^{\prime}$ direction on the current strap at $x=d$ and in the $x$ direction on the feeders. Also, $\nabla f$ provides the delt a function behavior for the current density in the appropriate locations.

The magnitude of the current flowing on the strap at $x=d$ is described by a surface current function, $K(y, z)$, that is continuous and defined throughout the periodic domain. Thus, the current density on this element is modeled by

$$
\vec{J}=K(y, z) \frac{\partial f(x, y, z)}{\partial x}(\cos \theta \hat{z}+\sin \theta \hat{y})+J_{x} \hat{x} .
$$

The current density on the feeders is chosen to be

$$
J_{x}=-K(y, z)\left[\cos \theta \frac{\partial f}{\partial z}+\sin \theta \frac{\partial f}{\partial y}\right] j_{x}(x)
$$


where the complex function, $j_{x}(x)$, describes the variation of current along the feeders. Constraining the value of $j_{x}(x=d)=1$ ensures continuity of the currents flowing from the main strap onto the feedes s at $z^{\prime}= \pm \beta / 2$. This constraint forces the delta function behavior at each end of the strap to be canceled analytically with the appropriate terms at the end of each feeder. A general expansion for $j_{x}$ gives

$$
j_{x}(x)=\sum_{p=-\infty}^{\infty} j_{p} \exp \left[i p \pi(x-d) / a_{w}\right]
$$

with

$$
\sum_{p=-\infty}^{\infty} j_{p}=1
$$

and

$$
j_{p}=\frac{1}{2 a_{w}} \int_{d-a_{w}}^{d+a_{w}} j_{x}(x) \exp \left[-i p \pi(x-d) / a_{w}\right] d x .
$$

The step behavior in the geometry function, $f$, restricts the use of $j_{x}$ to the range from $x=d$ to $x=d+a_{w}$.

The Fourier analysis for the geometry function is carried out by applying the example of Eq. (3) to Eq. (5). The integration is carried out in the $\left(x^{\prime}, y^{\prime}, z^{\prime}\right)$ coordinate system, where the step functions can be used to limit the range of integration. The result is

$$
\begin{aligned}
f^{m, n}(x) & =\exp \left\{-i\left[\left(m y_{c} / a\right)+\left(n z_{c} / R\right)\right]\right\} \frac{\Theta(x-d)}{\pi^{2} a R} \\
& \times \frac{\sin \{\alpha[(m / a) \cos \theta-(n / R) \sin \theta] / 2\}}{[(m / a) \cos \theta-(n / R) \sin \theta]} \\
& \times \frac{\sin \{\beta[(m / a) \sin \theta+(n / R) \cos \theta] / 2\}}{[(m / a) \sin \theta+(n / R) \cos \theta]}
\end{aligned}
$$

for $m, n \neq 0$, and

$$
f^{0,0}(x)=\frac{\alpha \beta}{4 \pi^{2} a R} \Theta(x-d) .
$$

Fourier components of the currents are obtained by using convolutions of $K$ with $f$. $K$ can be used to represent spatial variations in the driven of current that arise from finite phase velocity, e.g., $K\left(y^{\prime}, z\right)=\cos \left(\omega y^{\prime} / v_{\phi}\right)$. Using orthogonality of the basis functions, we find the Fourier components of $\vec{J}$ from Eq. (6):

$$
J^{m, n}=\sum_{m^{\prime}, n^{\prime}} K^{m^{\prime}, n^{\prime}} \frac{d f}{d x}{ }^{m-m^{\prime}, n-n^{\prime}}(\cos \theta \hat{z}+\sin \theta \hat{y})+J_{x}^{m, n} \hat{x} .
$$

When a finite number of Fourier modes are used, twice as many modes must be retained for the $f$ function as for the $K$ function to produce the correct $\vec{J}^{m, n}$ coefficients. In Fourier space, the feeder currents are

$$
\begin{aligned}
J_{x}^{m, n} \equiv & \mathcal{J}_{x}^{m, n} j_{x}(x)=-i \sum_{m^{\prime}, n^{\prime}} f^{m-m^{\prime}, n-n^{\prime}} K^{m^{\prime}, n^{\prime}} \\
& \times\left(\frac{m-m^{\prime}}{a} \sin \theta+\frac{n-n^{\prime}}{R} \cos \theta\right) j_{x}(x)
\end{aligned}
$$


The solution to Eq. (4) is obtained analytically for each Fourier mode by considering a specified current element to be located in Region I, as shown in Fig. 3. In this region, the solution is

$$
\begin{aligned}
E_{\nu \mathrm{I}}^{m, n}= & A_{\mathrm{I}}^{m, n} \exp \left[i k_{\perp}^{m, n}\left(x-d-a_{w}\right)\right] \\
& +B_{\mathrm{I}}^{m, n} \exp \left[-i k_{\perp}^{m, n}\left(x-d-a_{w}\right)\right] \\
& +\mathrm{P}_{v}^{m, n}(x)
\end{aligned}
$$

and

$$
\begin{aligned}
E_{x I}^{m, n}= & C_{I}^{m, n} \exp \left[i k_{\perp}^{m, n}\left(x-d-a_{w}\right)\right] \\
& +D_{I}^{m, n} \exp \left[-i k_{\perp}^{m, n}\left(x-d-a_{w}\right)\right] \\
& +P_{z}^{m, n}(x)
\end{aligned}
$$

where $P_{y}^{m, n}(x)$ and $P_{y}^{m, n}(x)$ are the particular solutions caused by any charge distributed along the feeder. The coefficients, $A_{1}^{m, n}, B_{I}^{m, n}, C_{I}^{m, n}$ and $D_{I}^{m, n}$, are determined by the boundary condition at $x=d+a_{w}$ and matching conditions at $x=d$. The homogeneous solution to Eq. (4) between the current element and the plasma, denoted as Region II, is given by

$$
\begin{gathered}
E_{\nu \mathrm{II}}^{m, n}=A_{\mathrm{II}}^{m, n} \exp \left(i k_{\perp}^{m, n} x\right)+B_{\mathrm{II}}^{m, n} \exp \left(-i k_{\perp}^{m, n} x\right) \\
E_{\mathrm{zII}}^{m, n}=C_{\mathrm{II}}^{m, n} \exp \left(i k_{\perp}^{m, n} x\right)+D_{\mathrm{II}}^{m, n} \exp \left(-i k_{\perp}^{m, n} x\right) .
\end{gathered}
$$

The coefficients, $A_{\mathrm{II}}^{m, n}, B_{\mathrm{II}}^{m, n}, C_{\mathrm{II}}^{m, n}$ and $D_{\mathrm{II}}^{m, n}$, are determined by applying the plasma surface impedance condition at $x=0$ and matching conditions at $x=d$. The matching conditions between Regions I and II are obtained by requiring continuity of $E_{y}$ and $E_{z}$ at $x=d$ and by integrating Eqs. (4a) and (4b) across an infinitesimally thin layer at $x=d$. The detailed values for these coefficients using various choices for feeder currents are given in Appendix A. 


\section{3-D MODELING AND 2-D BENCHMARK}

To model a 3-D antenna, the ORION-1D code was first used to generate a surface impedance for cold plasma. The plasma profile that was used, shown in Fig. 6, had a square root parabolic dependance from the magnetic axis to the separatrix and an exponential scrapeoff layer extending from the separatrix to the plasma surface. An absorber was used on the high field side of the magnetic axis so that no power was reflected from the conducting wall on the high field side of the tokamak. Other pertinent parameters for the plasma are given in Table 1.

TABLE I. PLASMA PARAMETERS FOR 3-D STUDY

\begin{tabular}{ll}
\hline Plasma parameters & $2.25 \mathrm{~m}$ \\
Major radius, $R$ & $0.53 \mathrm{~m}$ \\
Minor radius, $a$ & $0.48 \mathrm{~m}$ \\
Plasma minor radius, $a_{\mathrm{p}}$ & $8 \times 10^{18} \mathrm{~m}^{-3}$ \\
Central density, $n_{0}$ & $1.6 \times 10^{18} \mathrm{~m}^{-3}$ \\
Separatrix density, $n_{e}$ & $0.02 \mathrm{~m}$ \\
Exponential decay length & $0.05 \mathrm{~m}$ \\
Width of exponential region & $40 \mathrm{MHz}$ \\
Frequency, $\omega$ & 4 Tesla \\
Central magnetic field, $B_{0}$ & deuterium \\
Ion species & $-50 \mathrm{to} 50$ \\
Poloidal mode range & $-200 \mathrm{to} 200$ \\
Toroidal mode range & \\
Antenna module parameters & $0.02 \mathrm{~m}$ \\
Element width & $0.07 \mathrm{~m}$ \\
Strap width & $0.09 \mathrm{~m}$ \\
Strap height & $0.14 \mathrm{~m}$ \\
module width & $1.0 \mathrm{~m}$ \\
module height & \\
Distance from current strap & \\
to backwall, $a_{w}$ & $0.325 \mathrm{~m}$ \\
Distance from front of septum & \\
to backwall, $a$. & $0.3 \mathrm{~m}$ \\
\hline
\end{tabular}

The rf antenna to be modeled consisted of two antenna modules placed side by side in the toroidal direction producing a septum in between two current straps and sidewalls on either side. The current elements used by MAntIS to model the top portion of the structure are shown in Fig. 7 (note schematic for the main straps). Dimensions pertaining to each of the antenna modules are given in Table 1.

As shown in Fig. 8, two filaments were used to simulate the peaking of current near the edges of each main strap. The amplitude and phase of the currents carried by the filaments were varied to approximately satisfy the magnetic boundary conditions at the septum and sidewall locations. These currents were initially adjusted by visually inspecting the rf magnetic field at the locations where conducting boundary conditions are to be 
satisfied. An automated method based on the magnetic flux through the septum was then used for small changes in the currents. The filament locations were not varied. All currents for the main straps were kept in phase with one another (frequently referred to as monopole phasing).

The relative values of the currents modeling the septum and sidewalls (Fig. 8) were initially determined with a gap between the plasma and main straps, $d$, of $0.025 \mathrm{~m}$. This distribution was found to be relatively insensitive to $d$. For subsequent runs with different values of $d$, the currents carried by the septum and sidewalls were kept constant. The boundary conditions were then satisfied by adjusting the amplitude and phase of the currents carried by the main strap. To satisfy the boundary conditions for both the real and imaginary parts of the rf magnetic field, the phase of the currents in the main strap led the phase of the currents in the septum by slightly more than $\pi$ radians. The largest phase lead was roughly $\pi+\dot{v} .05$ radians for the case $d=0.015 \mathrm{~m}$ and was negligible for $d>0.05 \mathrm{~m}$. This additional phase delay appears to be caused by the complex plasma impedance.

The procedure for satisfying the boundary conditions was automated by integrating the $z$ component of the if magnetic field, $B_{z}$, at $y=0, z=0$ along the septum between $x=d$, and $x=d_{a}+0.3 \mathrm{~m}$. The current amplitude was adjusted until the real part of the integral of $B_{z}$ along this line was zero, and then the phase was adjusted to make the imaginary part of the integral of $B_{z}$ over the septum also go to zero. The norm used to determine zero was the amplitude of the integral of $B_{z}$ between the plasma $(x=0)$ and the septum $\left(x=d_{s}\right)$ at $y=0$ and $z=0$.

The validity of this procedure and the accuracy of the modeling was then benchmarked by considering the 2-D limit and comparing the results with those from the 2-D code, RANT. To model the 2-D limit, the filaments for the 3-D model were extended to the full periodic length in the poloidal direction. In this limit, the currents carried by the feeders overlap and completely cancel one another. This cancellation also occurs for the elements that are used to model the top and bottom of the antenna box (Fig. 7). In the 2-D benchmark case the results of the 3-D filament model and the 2-D model agreed for both real and imaginary power to within $10 \%$. This agreement is very good considering the different ways that the currents in the septum and sidewalls are calculated in the two models. In the MAntIS model, only three elements are used for modeling the septum and sidewalls, and these three elements were adjusted together to find the root for the flux througin the septum. (The distribution between the three was the same as that selected for the case with 1.0-m poloidal extent, shown in Fig. 8.) The 2-D RANT code calculates the currents in the sidewalls according to boundary conditions on the fields in a way that is self-consistent with the forced current in the main strap. The benchmark also shows that the impedance matrix for the plasma response in the two models is consistent even though calculated by two different methods-finite differences for ORION-1D and integration with a WKB outgoing boundary for RANT. Thus, we conclude that differences in results found for the 3-D model using MAntIS and those of the 2-D predictions using RANT arise from 3-D geometrical effects or the 3-D plasma response. 


\section{RESULTS}

For the first study of 3-D effects, constant current was maintained along all of the current elements, producing zero divergence for the driven currents (no driven charge in the source terms of the model). The poloidal extent of the antenna for the 3-D model was selected to be $1 \mathrm{~m}$, as described in Table 1 and Fig. 7 . The effect of the plasma on the currents in the septum and sidewalls was studied by varying the distance between the main current strap and the plasma in both the 3-D and 2-D models.

A good measure of the effect of the plasma on each model is obtained by summing all of the currents passing through the $y=0$ poloidal plane and the conducting backwall. The sum includes the current fiowing in the main strap, the septum and sidewalls, and the conducting backwall but not the plasma. This sum, normalized to the current carried in one of the main straps, is shown in Fig. 9 for both the 3-D and 2-D models. As shown in Fig. 9, the 2-D model predicts a significant value for the total current in the presence of plasma. Note that the plasma induces both a real and imaginary component in the return currents in the 2-D model. This total current in the 2-D limit becomes large as the plasma is brought near the main current elements and is referred to as "current robbing" by the plasma. As the figure indicates, the 3-D model for the finite-length antenna does not exhibit the current-robbing effect but did show the current-robbing effect when the antenna length was extended to the 2-D limit. For the 3-D model, a slight change (roughly 5\% over the range studied) in the ratio of currents in the main strap to the septum and sidewalls is observed, but this change is canceled by currents flowing in the conducting backwall. (The error bars for the 3-D data in Fig. 9 are an estimate of the error in calculating the return currents in the conducting backwall using a finite number of poloidal modes.) Thus, the 3-D model shows that the current-robbing effect is an artifact of the 2-D model.

The reason for the lack of current robbing in the finite-length, 3-D model is that the feeders, septum, and sidewalls of the antenna structure combine to provide a low-inductance path for return currents. This path prevents the plasma from participating in the circuit over the entire poloidal domain. The absence of both feeders and the top of the antenna box in the 2-D model permit currents (including those carried by the plasma) to return throngh the periodic boundary condition: a loop the entire distance around the plasma. This absence of a short to the backwall in the 2-D model permits the plasma to unrealistically affect the return currents in the septum and sidewalls.

The robbed current in the 2-D model can influence the spectrum of power launched in the toroidal direction. This difference between the 2-D and 3-D predictions for the toroidal power spectrum is illustrated in Fig. 10 by averaging over all poloidal mode numbers. A significant reduction in the loading is caused by the depression for small toroidal mode numbers for the 3-D model as compared with the 2-D model.

Another 3-D effect that reduces loading arises from the increased density required to propagate modes with a nonzero poloidal mode number. This effect tends to be important when the plasma density in the exponential region (see Fig. 6) is near the cutoff density for the fast wave. For the parameters in Table 1, a significant portion of the antenna spectrum can be cut off by the plasma. The effect is demonstrated in Fig. 11 which illustrates the loading per unit length versus the length of the antenna. (The current distribution was not varied during the calculation, and elements representing the top and bottom of the antenna box were removed). For the longest antenna, only the $m=0$ mode is excited, while for the 
shorter lengths a broader range of poloidal modes is excited.

Loading calculations made by the 2-D and 3-D models are summarized in Fig. 12 for the case of infinite phase velocity, $v_{\phi}$. For $v_{\phi}=\infty$, the distribution of current along the current elements was a constant $(\nabla \cdot J=0)$, thereby eliminating any source term arising from charge in the 3-D model. Note that the 2-D model always satisfies $\nabla \cdot J=0$ for the antenna currents, because $m=0$ and only the $y$ component of the electric field is retained. The real part of the loading from the 2-D calculation has been reduced by a factor of 0.65 to account for end effects [9]. (Such a factor is always used for the 2-D loading predictions based on vacuum measurements of the magnetic field near the antennas.) Because the currents modeling the antenna are divergence-free, the stored energy in the electric fields of the system is small and the imaginary part of the loading per strap can be approximately estimated from $\left(P_{i}\right) / 2 \approx \omega L I^{2} / 2$, where $L$ is the inductance and $P_{i}$ is the circulating (imaginary) power. The power is divided by 2 to obtain an average value per strap. Note that both the real loading and inductance are overestimated in the 2-D model. The 3-D results are near the experimentally measured values for the inductance. 


\section{DISCUSSION}

Several 3-D effects are found to significantly influence estimates used in rf antenna designs. One important effect is the absence of a significant change in the distribution of currents flowing in the septum and sidewalls when plasma parameters are varied. In 2-D models, the only return path available is along the entire poloidal extent of the device. In the 3-D model, the feeders provide a return path to the backwall with a much lower inductance than a return path covering the entire poloidal extent of the device. Thus, the plasma does not significantly "rob" current from the antenna structure when the feeders are included, and the sidewalls remain strongly coupled to the main strap despite the proximity of the plasma. This 3-D phenomenon affects the launched power spectrum by depressing the power spectrum at low toroidal mode numbers, as shown in Fig. 10.

Another significant 3-D effect can occur because of the variation of the plasma impedance matrix with poloidal mode number. Modes with $m \neq 0$ (nonzero poloidal modes) require a higher density to propagate into the plasma than the $m=0$ mode for any chosen toroidal mode. Thus, the reduced loading for nonzero poloidal mode numbers can significantly reduce the total loading, as shown in Fig. 11. This effect tends to be important when the plasma edge derisity is near cutoff for a significant portion of the driven antenna spectrum. The effect is also more significant for devices with small minor radius, because the poloidal structure of the antenna must be constructed from a discrete set of modes having larger poloidal wave numbers, $m / a$. The 3-D model also describes the inductance of the rf antenna better than the 2-D model as shown in Fig. 12.

In conclusion, 3-D effects can lead to results for if antenna loading calculations that differ from 2-D models. These differences can be as large as a factor of 2 for some plasma conditions. Three-dimensional effects can be important in applications using closely spaced, phased arrays of antennas, and when the plasma in the scrapeoff layer is near the fast wave cutoff for a significant portion of the poloidal antenna spectrum. 


\section{APPENDIX: A}

For each basic current element, the current-free region between the plasma-vacuum interface at $x=0$ and the current strap at $x=d$ is denoted Region II (see Fig. 3). The region between the strap at $x=d$ and the backwall at $x=d+a_{w}$ is denoted Region I. A specified electric field is applied for the boundary condition at the backwall. For example, $E_{y}^{m, n}\left(x=d+a_{w}\right)=0$ and $E_{z}^{m, n}\left(x=d+a_{w}\right)=0$ is the boundary condition for a perfectly conducting backwall.

At the interface between Regions I and II, the delta function, $\delta(x-d)$, arising from $\partial f / \partial x$ in Eq. $(6 a)$ is treated analytically. Equations $(4 a)$ and $(4 b)$ are integrated across the infinitesimal boundary at $x=d$ while maintaining continuity for both the $E_{y}^{m, n}$ and $E_{z}^{m, n}$ components. ( $E_{x}^{m, n}$ can be discontinuous at $x=d$.) In Region $I$, the delta functions arising from $\partial f / \partial y$ and $\partial f / \partial z$ in Eq. (6b) are true discontinuities only in the limit that an infinite number of Fourier modes is considered. Thus, the scale length of resolution for the feeders and the ends of the main strap depends upon the largest Fourier harmonic retained in the calculation.

With Eqs. (7a) and (9b), the solutions to Eqs. (4a) and (4b) in Region I are

$$
\begin{aligned}
E_{\mathrm{yI}}^{m, n}= & A_{\mathrm{I}}^{m, n} \exp \left[i k_{\perp}^{m, n}\left(x-d-a_{w}\right)\right] \\
& +B_{\mathrm{I}}^{m, n} \exp \left[-i k_{\perp}^{m, n}\left(x-d-a_{w}\right)\right] \\
& +\mathrm{P}_{y}^{m, n}(x) \\
E_{\mathrm{zI}}^{m, n}= & C_{I}^{m, n} \exp \left[i k_{\perp}^{m, n}\left(x-d-a_{w}\right)\right] \\
& +D_{I}^{m, n} \exp \left[-i k_{\perp}^{m, n}\left(x-d-a_{w}\right)\right] \\
& +\mathrm{P}_{z}^{m, n}(x)
\end{aligned}
$$

where

$$
\mathrm{P}_{y}^{m, n}(x)=\frac{i \mu_{0} \pi c^{2}}{w a_{w}} \frac{m}{a} \mathcal{J}_{x}^{m, n} \sum_{p=-\infty}^{\infty} \frac{p j_{p} \exp \left[i p \pi(x-d) / a_{w}\right]}{k_{\perp}^{2 m, n}-p^{2} \pi^{2} / a_{w}^{2}}
$$

and

$$
\mathrm{P}_{z}^{m, n}(x)=\frac{i \mu_{0} \pi c^{2}}{\omega a_{w}} \frac{n}{R} \mathcal{J}_{x}^{m, n} \sum_{p=-\infty}^{\infty} \frac{p j_{p} \exp \left[i p \pi(x-d) / a_{w}\right]}{k_{\perp}^{2 m, n}-p^{2} \pi^{2} / a_{w}^{2}}
$$

Using the notation $E_{\mathrm{yI}}^{m, n}\left(x=d+a_{w}\right)$ to represent the specified field at the boundary, $x=d+a_{w}$, gives

$$
B_{\mathrm{I}}^{m, n}=E_{\nu \mathrm{I}}^{m, n}\left(x=d+a_{w}\right)-A_{\mathrm{I}}^{m, n}-\frac{i \mu_{0} \pi c^{2}}{\omega a_{w}} \frac{m}{a} \mathcal{J}_{x}^{m, n} \sum_{p=-\infty}^{\infty} \frac{p(-1)^{p} j_{p}}{k_{\perp}^{2 m, n}-p^{2} \pi^{2} / a_{w}^{2}}
$$

and

$$
D_{\mathrm{I}}^{m, n}=E_{\mathrm{zI}}^{m, n}\left(x=d+a_{w}\right)-C_{\mathrm{I}}^{m, n}-\frac{i \mu_{0} \pi c^{2}}{\omega a_{w}} \frac{n}{R} \mathcal{J}_{x}^{m, n} \sum_{p=-\infty}^{\infty} \frac{p(-1)^{p} j_{p}}{k_{\perp}^{2 m, n}-p^{2} \pi^{2} / a_{w}^{2}} .
$$


In Region II, the solutions to Eqs. (4a) and (4b) are

$$
\begin{gathered}
E_{y I I}^{m, n}=A_{\mathrm{II}}^{m, n} \exp \left(i k_{\perp}^{m, n} x\right)+B_{\mathrm{II}}^{m, n} \exp \left(-i k_{\perp}^{m, n} x\right) \\
E_{\mathrm{zII}}^{m, n}=C_{\mathrm{II}}^{m, n} \exp \left(i k_{\perp}^{m, n} x\right)+D_{\mathrm{II}}^{m, n} \exp \left(-i k_{\perp}^{m, n} x\right) .
\end{gathered}
$$

Integration of Eqs. (4a) and (4b) across the $x=d$ location yields the jump condition for the derivatives at $x=d$ :

$$
\left.\frac{d E_{\mathrm{yI}}^{m, n}}{d x}\right|_{x=d}-\left.\frac{d E_{y I I}^{m, n}}{d x}\right|_{x=d}=-i \omega \mu_{0} \mathcal{K}_{y}^{m, n}
$$

where

$$
\mathcal{K}_{\nu}^{m, n} \equiv \sum_{m^{\prime}, n^{\prime}} f^{m-m^{\prime}, n-n^{\prime}} K^{m^{\prime}, n^{\prime}}\left[\left(1-\frac{c^{2}}{\omega^{2}} \frac{m m^{\prime}}{a^{2}}\right) \sin \theta-\frac{c^{2}}{\omega^{2}} \frac{m n^{\prime}}{a R} \cos \theta\right]
$$

and

$$
\left.\frac{d E_{z I}^{m, n}}{d x}\right|_{x=d}-\left.\frac{d E_{z I I}^{m, n}}{d x}\right|_{x=d}=-i \omega \mu_{0} \mathcal{K}_{x}^{m, n}
$$

where

$$
K_{z}^{m, n} \equiv \sum_{m^{\prime}, n^{\prime}} f^{m-m^{\prime}, n-n^{\prime}} K^{m^{\prime}, n^{\prime}}\left[\left(1-\frac{c^{2}}{\omega^{2}} \frac{n n^{\prime}}{R^{2}}\right) \cos \theta-\frac{c^{2}}{\omega^{2}} \frac{n m^{\prime}}{a R} \sin \theta\right] .
$$

The surface terms, $\mathcal{K}_{y}^{m, n}$ and $\mathcal{K}_{z}^{m, n}$, include both current and charge sources for the fields.

Differentiating Eqs. (A1) and (A2) for use in Eq. (A3), we find that the jump condition at $x=d$ becomes

$$
\begin{gathered}
i k_{\perp}^{m, n}\left[A_{\mathrm{II}}^{m, n} \exp \left(i k_{\perp}^{m, n} d\right)-B_{\mathrm{II}}^{m, n} \exp \left(-i k_{\perp}^{m, n} d\right)\right]=i k_{\perp}^{m, n}\left[A_{1}^{m, n} \exp \left(-i k_{\perp}^{m, n} a_{w}\right)\right. \\
\left.\quad-B_{1}^{m, n} \exp \left(i k_{\perp}^{m, n} a_{w}\right)\right]+i \omega \mu_{0} \mathcal{K}_{\nu}^{m, n}+\left.\frac{d \mathrm{P}_{y}^{m, n}}{d x}\right|_{x=d} \\
i k_{\perp}^{m, n}\left[C_{\mathrm{II}}^{m, n} \exp \left(i k_{\perp}^{m, n} d\right)-D_{\mathrm{II}}^{m, n} \exp \left(-i k_{\perp}^{m, n} d\right)\right]=i k_{\perp}^{m, n}\left[C_{\mathrm{I}}^{m, n} \exp \left(-i k_{\perp}^{m, n} a_{w}\right)\right. \\
\left.-D_{\mathrm{I}}^{m, n} \exp \left(i k_{\perp}^{m, n} a_{w}\right)\right]+i \omega \mu_{0} \mathcal{K}_{z}^{m, n}+\left.\frac{d \mathrm{P}_{z}^{m, n}}{d x}\right|_{x=d},
\end{gathered}
$$

and the match condition at $x=d$ is obtained using Eqs. (A1) and (A2) to give

$$
\begin{gathered}
A_{\mathrm{II}}^{m, n} \exp \left(i k_{\perp}^{m, n} d\right)+B_{\mathrm{II}}^{m, n} \exp \left(-i k_{\perp}^{m, n} d\right)=A_{\mathrm{I}}^{m, n} \exp \left(-i k_{\perp}^{m, n} a_{w}\right) \\
+B_{\mathrm{I}}^{m, n} \exp \left(i k_{\perp}^{m, n} a_{w}\right)+\mathrm{P}_{y}^{m, n}(x=d)
\end{gathered}
$$

and

$$
\begin{gathered}
C_{\mathrm{II}}^{m, n} \exp \left(i k_{\perp}^{m, n} d\right)+D_{\mathrm{II}}^{m, n} \exp \left(-i k_{\perp}^{m, n} d\right)=C_{\mathrm{I}}^{m, n} \exp \left(-i k_{\perp}^{m, n} a_{w}\right) \\
+D_{\mathrm{I}}^{m, n} \exp \left(i k_{\perp}^{m, n} a_{w}\right)+\mathrm{P}_{z}^{m, n}(x=d)
\end{gathered}
$$


Using Eq. (A4), we can write the Region II coefficients in terms of $A_{1}^{m, n}$ and $C_{1}^{m, n}$ so that Eq. (A2) becomes

$$
\begin{aligned}
E_{y I I}^{m, n} & =A_{\perp}^{m, n}\left\{\exp \left[i k_{\perp}^{m, n}\left(x-a_{w}-d\right)\right]-\exp \left[-i k_{\perp}^{m, n}\left(x-a_{w}-d\right)\right]\right\} \\
& +\frac{\omega \mu_{0}}{2 k_{\perp}^{m, n}} K_{y}^{m, n}\left\{\exp \left[i k_{\perp}^{m, n}(x-d)\right]-\exp \left[-i k_{\perp}^{m, n}(x-d)\right]\right\} \\
& +E_{y}^{m, n}\left(x=d+a_{w}\right) \exp \left[-i k_{\perp}^{m, n}\left(x-a_{w}-d\right)\right] \\
& +\frac{i \mu_{0} \pi c^{2}}{\omega a_{w}} \frac{m}{a} J_{x}^{m, n} \sum_{p=-\infty}^{\infty} \frac{p j_{p}}{k_{\perp}^{2 m, n}-p^{2} \pi^{2} / a_{w}^{2}} \\
& \times\left\{\frac{1}{2}\left(\frac{p \pi}{k_{\perp}^{m, n} a_{w}}+1\right) \exp \left[i k_{\perp}^{m, n}(x-d)\right]\right. \\
& \left.-\frac{1}{2}\left(\frac{p \pi}{k_{\perp}^{m, n} a_{w}}-1\right) \exp \left[-i k_{\perp}^{m, n}(x-d)\right]-(-1)^{p} \exp \left[-i k_{\perp}^{m, n}\left(x-d-a_{w}\right)\right]\right\} \\
E_{x I I}^{m, n} & =C_{1}^{m, n}\left\{\exp \left[i k_{\perp}^{m, n}\left(x-a_{w}-d\right)\right]-\exp \left[-i k_{\perp}^{m, n}\left(x-a_{w}-d\right)\right]\right\} \\
& +\frac{\omega \mu_{0}}{2 k_{\perp}^{m, n}} \mathcal{K}_{z}^{m, n}\left\{\exp \left[i k_{\perp}^{m, n}(x-d)\right]-\exp \left[-i k_{\perp}^{m, n}(x-d)\right]\right\} \\
& +E_{z}^{m, n}\left(x=d+a_{w}\right) \exp \left[-i k_{\perp}^{m, n}\left(x-a_{w}-d\right)\right] \\
& +\frac{i \mu_{0} \pi c^{2}}{\omega a_{w}} \frac{n}{R} \mathcal{J}_{x}^{m, n} \sum_{p=-\infty}^{\infty} \frac{p j_{p}}{k_{\perp}^{2 m, n}-p^{2} \pi^{2} / a_{w}^{2}} \\
& \times\left\{\frac{1}{2}\left(\frac{p \pi}{k_{\perp}^{m, n} a_{w}}+1\right) \exp \left[i k_{\perp}^{m, n}(x-d)\right]\right. \\
& \left.-\frac{1}{2}\left(\frac{p \pi}{k_{\perp}^{m, n} a_{w}}-1\right) \exp \left[-i k_{\perp}^{m, n}(x-d)\right]-(-1)^{p} \exp \left[-i k_{\perp}^{m, n}\left(x-d-a_{w}\right)\right]\right\}
\end{aligned}
$$

The last two equations for the Region I coefficients are obtained by considering the match between the vacuum region (Region II) and the plasma. The match is taken to be in the vacuum just outside the plasma surface, so that if no source currents pass through the interface between the vacuum region and the plasma surface $[\vec{J}(x=0)=0]$, then continuity of the magnetic field implies that the matching condition can take the form of an impedance matrix [4]. The impedance match at $x=0$ is written with the assumption that toroidal and poloidal modes are not coupled (periodic slab approximation), giving

$$
\begin{aligned}
& E_{\nu}^{m, n}(x=0)=\frac{1}{\mu_{0}}\left[\mathcal{Z}_{11}^{m, n} B_{y}^{m, n}+\mathcal{Z}_{12}^{m, n} B_{z}^{m, n}\right]_{x=0} \\
& E_{z}^{m, n}(x=0)=\frac{1}{\mu_{0}}\left[\mathcal{Z}_{21}^{m, n} B_{y}^{m, n}+\mathcal{Z}_{22}^{m, n} B_{z}^{m, n}\right]_{x=0},
\end{aligned}
$$

where the $\mathcal{Z}_{i j}^{m, n}$ coefficients of the impedance matrix are calculated as described in Appendix $B$ and the notation $[\ldots]_{x=0}$ indicates that all field components inside the brackets 
are to be evaluated at $x=0$. The tangential components of $\nabla \times \vec{E}=i \omega \vec{B}$ in Fourier space give

$$
\begin{gathered}
{\left[i \omega B_{y}^{m, n}=\frac{i n}{R} E_{x}^{m, n}-\frac{d E_{z}^{m, n}}{d x}\right]_{x=0}} \\
{\left[i \omega B_{z}^{m, n}=\frac{d E_{y}^{m, n}}{d x}-\frac{i m}{a} E_{x}^{m, n}\right]_{x=0} .}
\end{gathered}
$$

Thus, if the impedance matrix is known (see Appendix B), then Eq. (A5) can be used to calculate $d E_{y}^{m, n} / d x$ and $d E_{z}^{m, n} / d x$ in Region II at $x=0$. These derivatives at $x=0$ are then used with Eq. $(4 \mathrm{c})$ to eliminate $E_{x}^{m, n}(x=0)$ in Eqs. (A7a) and (A7b) with the result used to eliminate the magnetic field components in Eq. (A6). These steps result in two equations that relate the two unknown Region I coefficients, $A_{\mathrm{I}}^{m, n}$ and $C_{\mathrm{I}}^{\mathrm{m}, n}$, by using Eq. (A5) to evaluate $E_{y}^{m, n}$ and $E_{z}^{m, n}$ at $x=0$.

Specific solutions for $A_{\mathrm{I}}^{m, n}$ and $C_{\mathrm{I}}^{m, n}$ for two feeder options have been tested in MAntIS and are given below. The first option includes currents that are constant and continuous along the feeder. The second feeder option permits both standing wave and traveling wave currents with wavelength $2 a_{w}$ to flow on the feeder elements. This option has been used primarily for folded waveguide modeling $[7,8]$.

Two combinations of feeder type and backwall boundary have been tested in MAntIS for two different types of modeling. Both combinations satisfy Eq. (7b) (see Sect. 2). The first combination has constant current along each feeder, $j_{0}=1$ and $j_{p \neq 0}=0$, with boundary conditions for a conducting backwall:

$$
E_{y 1}^{m, n}\left(x=d+a_{w}\right)=0
$$

and

$$
E_{x \mathrm{l}}^{m, n}\left(x=d+a_{w}\right)=0
$$

This combination has been used for typical loop antenna modeling, as demonstrated in this paper.

The second combination has both standing and traveling waves for the current along the feeder such that $j_{1}=(\zeta+1 / 2), j_{-1}=-(\zeta-1 / 2)$, and $j_{p \neq \pm 1}=0$. The boundary conditions used with this feeder type are

$$
\begin{gathered}
E_{y \mathrm{I}}^{m, n}\left(x=d+a_{w}\right)=\frac{i \mu_{0} \pi c^{2}}{\omega a_{w}} \frac{m}{a} \mathcal{J}_{x}^{m, n} \frac{-2 \zeta}{\left(k_{\perp}^{2 m, n}-\pi^{2} / a_{w}^{2}\right)} \\
E_{\mathrm{z} \mathrm{I}}^{m, n}\left(x=d+a_{w}\right)=\frac{i \mu_{0} \pi c^{2}}{\omega a_{w}} \frac{n}{R} \mathcal{J}_{x}^{m, n} \frac{-2 \zeta}{\left(k_{\perp}^{2 m, n}-\pi^{2} / a_{w}^{2}\right)}
\end{gathered}
$$

where $\mathcal{J}_{x}^{m, n}$ is defined by Eq. (9b), and $\zeta$ is a traveling wave parameter. This boundary condition is equivalent to a conducting backwall for standing waves where $\zeta=0$, but it permits power carried by the traveling wave to flow through the backwall when $\zeta \neq 0$. This combination has been used to model folded waveguide antenna structures $[7,8]$ primarily for $\zeta=0$. 
Noting that Eq. (A8) is a simplification of Eq. (A9) obtained by taking $\zeta=0$ and eliminating the particular solutions, the fields in Region II are given by

$$
\begin{aligned}
E_{y I I}^{m, n} & =A_{I}^{m, n}\left\{\exp \left[i k_{\perp}^{m, n}\left(x-a_{w}-d\right)\right]-\exp \left[-i k_{\perp}^{m, n}\left(x-a_{w}-d\right)\right]\right\} \\
& +\frac{\omega \mu_{0}}{2 k_{\perp}^{m, n}}\left(\mathcal{K}_{y}^{m, n}+\frac{\pi}{a_{w} \omega \mu_{0}} \mathcal{P}_{\nu}^{m, n}\right)\left\{\exp \left[i k_{\perp}^{m, n}(x-d)\right]-\exp \left[-i k_{\perp}^{m, n}(x-d)\right]\right\} \\
& +\mathcal{P}_{\nu}^{m, n} \zeta\left\{\exp \left[i k_{\perp}^{m, n}(x-d)\right]+\exp \left[-i k_{\perp}^{m, n}(x-d)\right]\right\} \\
E_{z I I}^{m, n} & =C_{I}^{m, n}\left\{\exp \left[i k_{\perp}^{m, n}\left(x-a_{w}-d\right)\right]-\exp \left[-i k_{\perp}^{m, n}\left(x-a_{w}-d\right)\right]\right\} \\
& +\frac{\omega \mu_{0}}{2 k_{\perp}^{m, n}}\left(\mathcal{K}_{z}^{m, n}+\frac{\pi}{a_{w} \omega \mu_{0}} \mathcal{P}_{z}^{m, n}\right)\left\{\exp \left[i k_{\perp}^{m, n}(x-d)\right]-\exp \left[-i k_{\perp}^{m, n}(x-d)\right]\right\} \\
& +\mathcal{P}_{z}^{m, n} \zeta\left\{\exp \left[i k_{\perp}^{m, n}(x-d)\right]+\exp \left[-i k_{\perp}^{m, n}(x-d)\right]\right\},
\end{aligned}
$$

where

$$
\mathcal{P}_{y}^{m, n} \equiv \frac{\mu_{0} \pi c^{2} / \omega a_{w}}{k_{\perp}^{2 m, n}-\pi^{2} / a_{w}^{2}} \frac{m}{a} \sum_{m^{\prime}, n^{\prime}} K^{m^{\prime}, n^{\prime}} f^{m-m^{\prime}, n-n^{\prime}}\left(\frac{m-m^{\prime}}{a} \sin \theta+\frac{n-n^{\prime}}{R} \cos \theta\right)
$$

and

$$
\mathcal{P}_{z}^{m, n} \equiv \frac{n a}{m R} \mathcal{P}_{y}^{m, n}
$$

The solutions for the Region I coefficients, $A_{\mathrm{I}}^{m, n}$ and $C_{\mathrm{I}}^{m, n}$, are compactly written as

$$
\begin{aligned}
& A_{I}^{m, n}=\Gamma_{a y}^{m, n} K_{y_{e \pi}}^{m, n}+\Gamma_{a z}^{m, n} K_{z_{e f}}^{m, n}-\zeta\left(\Gamma_{a p y}^{m, n} \mathcal{P}_{y}^{m, n}+\Gamma_{a p z}^{m, n} \mathcal{P}_{z}^{m, n}\right) \\
& C_{I}^{m, n}=\Gamma_{c y}^{m, n} K_{y_{e f}}^{m, n}+\Gamma_{c z}^{m, n} K_{z_{e f}}^{m, n}-\zeta\left(\Gamma_{c p y}^{m, n} \mathcal{P}_{y}^{m, n}+\Gamma_{c p z}^{m, n} \mathcal{P}_{z}^{m, n}\right)
\end{aligned}
$$

where

$$
\begin{aligned}
& \left.i \omega \mu_{0} K_{\nu_{e} n}^{m, n} \equiv \frac{d \mathrm{P}_{y}^{m, n}}{d x}\right|_{x=d}+i \omega \mu_{0} K_{\nu}^{m, n} \\
& \left.i \omega \mu_{0} K_{x_{e}, n}^{m, n} \equiv \frac{d \mathrm{P}_{z}^{m, n}}{d x}\right|_{x=d}+i \omega \mu_{0} K_{z}^{m, n}
\end{aligned}
$$

and

$$
\begin{aligned}
\Gamma_{a y}^{m, n} & \equiv \frac{\omega \mu_{0}}{2 k_{+}^{m, n}}\left[\left(\psi_{-}^{m, n}-\alpha_{1}^{m, n} \psi_{+}^{m, n}\right)\left(-\phi_{-}^{m, n}+\beta_{2}^{m, n} \phi_{+}^{m, n}\right)+\alpha_{2}^{m, n} \beta_{1}^{m, n} \psi_{+}^{m, n} \phi_{+}^{m, n}\right] / \Gamma_{d}^{m, n} \\
\Gamma_{a z}^{m, n} & \equiv \frac{\omega \mu_{0}}{2 k_{\perp}^{m, n}}\left[\beta_{1}^{m, n}\left(\psi_{+}^{m, n} \phi_{-}^{m, n}-\psi_{-}^{m, n} \phi_{+}^{m, n}\right)\right] / \Gamma_{d}^{m, n} \\
\Gamma_{a p y}^{m, n} & \equiv\left[\left(\alpha_{1}^{m, n} \psi_{-}^{m, n}-\psi_{+}^{m, n}\right)\left(\phi_{-}^{m, n}-\beta_{2}^{m, n} \phi_{+}^{m, n}\right)+\alpha_{2}^{m, n} \psi_{-}^{m, n} \beta_{1}^{m, n} \phi_{+}^{m, n}\right] / \Gamma_{d}^{m, n} \\
\Gamma_{a p z}^{m, n} & \equiv\left[\beta_{1}^{m, n}\left(\psi_{-}^{m, n} \phi_{-}^{m, n}-\psi_{+}^{m, n} \phi_{+}^{m, n}\right)\right] / \Gamma_{d}^{m, n} \\
\Gamma_{c y}^{m, n} & \equiv \frac{\omega \mu_{0}}{2 k_{+}^{m, n}}\left[\alpha_{2}^{m, n}\left(\psi_{+}^{m, n} \phi_{-}^{m, n}-\phi_{+}^{m, n} \psi_{-}^{m, n}\right)\right] / \Gamma_{d}^{m, n} \\
\Gamma_{c z}^{m, n} & \equiv \frac{\omega \mu_{0}}{2 k_{\perp}^{m, n}}\left[\left(\beta_{2}^{m, n} \psi_{+}^{m, n}-\psi_{-}^{m, n}\right)\left(\phi_{-}^{m, n}-\alpha_{1}^{m, n} \phi_{+}^{m, n}\right)+\beta_{1}^{m, n} \psi_{+}^{m, n} \alpha_{2}^{m, n} \phi_{+}^{m, n}\right] / \Gamma_{d}^{m, n} \\
\Gamma_{c p y}^{m, n} & \equiv\left[\alpha_{2}^{m, n}\left(\phi_{-}^{m, n} \psi_{-}^{m, n}-\phi_{+}^{m, n} \psi_{+}^{m, n}\right)\right] / \Gamma_{d}^{m, n} \\
\Gamma_{c p z}^{m, n} & \equiv\left[\left(\beta_{2}^{m, n} \psi_{-}^{m, n}-\psi_{+}^{m, n}\right)\left(\phi_{-}^{m, n}-\alpha_{1}^{m, n} \phi_{+}^{m, n}\right)+\beta_{1}^{m, n} \psi_{-}^{m, n} \alpha_{2}^{m, n} \phi_{+}^{m, n}\right] / \Gamma_{d}^{m, n}
\end{aligned}
$$


with

$$
\begin{aligned}
\Gamma_{d}^{m, n} & \equiv\left(-\phi_{-}^{m, n}+\alpha_{1}^{m, n} \phi_{+}^{m, n}\right)\left(-\phi_{-}^{m, n}+\beta_{2}^{m, n} \phi_{+}^{m, n}\right)-\alpha_{2}^{m, n} \beta_{1}^{m, n}\left(\phi_{+}^{m, n}\right)^{2} \\
\alpha_{1}^{m, n} & \equiv \frac{1}{\omega \mu_{0} k_{\perp}^{m, n}}\left[\mathcal{Z}_{11}^{m, n}\left(\frac{m n}{a R}\right)-\left(\frac{w^{2}}{c^{2}}-\mathcal{Z}_{12}^{m, n} \frac{n^{2}}{R^{2}}\right)\right] \\
\beta_{1}^{m, n} & \equiv \frac{1}{\omega \mu_{0} k_{\perp}^{m, n}}\left[\mathcal{Z}_{11}^{m, n}\left(\frac{w^{2}}{c^{2}}-\frac{m^{2}}{a^{2}}\right)-\mathcal{Z}_{12}^{m, n}\left(\frac{m n}{a R}\right)\right] \\
\alpha_{2}^{m, n} & \equiv \frac{1}{\omega \mu_{0} k_{\perp}^{m, n}}\left[\mathcal{Z}_{21}^{m, n}\left(\frac{m n}{a R}\right)-\mathcal{Z}_{22}^{m, n}\left(\frac{w^{2}}{c^{2}}-\frac{n^{2}}{R^{2}}\right)\right] \\
\beta_{2}^{m, n} & \equiv \frac{1}{w \mu_{0} k_{\perp}^{m, n}}\left[\mathcal{Z}_{21}^{m, n}\left(\frac{w^{2}}{c^{2}}-\frac{m^{2}}{a^{2}}\right)-\mathcal{Z}_{22}^{m, n}\left(\frac{m n}{a R}\right)\right] \\
\phi_{ \pm}^{m, n} & \equiv \exp \left[i k_{\perp}^{m, n}\left(a_{w}+d\right)\right] \pm \exp \left[-i k_{\perp}^{m, n}\left(a_{w}+d\right)\right] \\
\psi_{ \pm}^{m, n} & \equiv \exp \left(i k_{\perp}^{m, n} d\right) \pm \exp \left(-i k_{\perp}^{m, n} d\right) .
\end{aligned}
$$

In terms of these Region I coefficients, the electric fields in Region I along with the particular solutions for existing feeder options are given by Eq. (A1), and the fields in Region II are given by Eq. (A5). 


\section{APPENDIX: B}

The calculation of the impedance matrix for the plasma-vacuum interface is obtained by using the ORION-1D code [5]. ORION-1D models a slab plasma using Fourier analysis in the direction of the static magnetic field and in the direction orthogonal to both pressure gradients and the static magnetic field. Periodicity conditions for the directions that are Fourier-analyzed are chosen to represent the poloidal and toroidal directions shown in Fig. 1. The equations solved are the three components of

$$
-\nabla \times \nabla \times \vec{E}+\frac{\omega^{2}}{c^{2}} \vec{E}+i \omega \mu_{0} \sum_{s} \overrightarrow{J_{s}}=\vec{S}
$$

where the summation is over the plasma species, $\mathbf{s}$, and $\vec{S}$ is an external source term. The plasma current density terms, $\vec{J}_{\text {, }}$, are calculated using second-order finite temperature corrections to the warm plasma dielectric tensor. A sixth-order finite difference technique is used to solve Eq. (B1) in the direction of the plasma density and temperature gradients. The numerical implementation used to calculate the impedance matrix is described below.

Faraday's law, $\nabla \times \vec{E}=i \omega \vec{B}$, applies at all finite difference nodes regardless of the presence of plasma currents. With a conservative finite difference scheme, the numerical representation of Faraday's law is

$$
\begin{gathered}
i k_{y} E_{z N}^{m, n}-i k_{z} E_{y N}^{m, n}=i \omega B_{x N}^{m, n} \\
i k_{z} E_{x N-1}^{m, n}-\left(E_{z N}^{m, n}-E_{z N-1}^{m, n}\right) / \Delta=i \omega B_{y N-1}^{m, n} \\
\left(E_{y N}^{m, n}-E_{y N-1}^{m, n}\right) / \Delta-i k_{y} E_{x N-1}^{m, n}=i \omega B_{z N-1}^{m, n},
\end{gathered}
$$

where $k_{y}=m / a$ and $k_{z}=n / R$ for periodic boundaries in the $y$ and $z$ directions. In Eq. (B2), $N$ refers to the numerical nodes just on the vacuum side of the plasma-vacuum boundary, and $\Delta$ is the discrete step size in the $x$ direction. Field values at these last two locations (separated by $\Delta / 2$ ) represent the numerical resolution of the plasma-vacuum boundary. The correspondences between field values and node location are shown in Fig. 13 and are such that the numerical implementation of Stokes's theorem and the divergence theorem are satisfied. The remaining Maxwell's equation is written for this node as

$$
\begin{gathered}
i k_{y} B_{z N}^{m, n}-i k_{z} B_{y N}^{m, n}=\mu_{0} J_{x N}^{m, n}-\frac{i \omega}{c^{2}} E_{x N}^{m, n} \\
i k_{x} B_{x N}^{m, n}-\left(B_{z N}^{m, n}-B_{z N-1}^{m, n}\right) i \Delta=\mu_{0} J_{y N}^{m, n}-\frac{i \omega}{c^{2}} E_{y N}^{m, n} \\
\left(B_{y N}^{m, n}-B_{y N-1}^{m, n}\right) / \Delta-i k_{y} B_{x N}^{m, n}=\mu_{0} J_{z N}^{m, n}-\frac{i \omega}{c^{2}} E_{z N}^{m, n}
\end{gathered}
$$

Using Eq. (B2) to eliminate $B_{x N}^{m, n}, B_{y N-1}^{m, n}$, and $B_{z N-1}^{m, n}$ in Eq. (B3) and considering $B_{y N}^{m, n}$ and $B_{2 N}^{m, n}$ to be source terms, we obtain the numerical boundary equations at the impedance match location $(x=0)$ :

$$
E_{x N}^{m, n}=\frac{-i c^{2}}{\omega}\left(\mu_{0} J_{x N}^{m, n}-i k_{y} B_{z N}^{m, n}+i k_{y} B_{y N}^{m, n}\right)
$$




$$
\begin{aligned}
& \frac{i c^{2}}{\omega^{2}} \frac{k_{y}}{\Delta} E_{x N-1}^{m, n}+\frac{c^{2}}{\omega^{2}} \frac{E_{y N-1}^{m, n}}{\Delta^{2}}+\frac{c^{2}}{\omega^{2}} k_{\nu} k_{z} E_{z N}^{m, n}+\left[1-\frac{c^{2}}{\omega^{2}}\left(k_{z}^{2}+1 / \Delta^{2}\right)\right] E_{y N}^{m, n} \\
& =\frac{-i c^{2}}{\omega} \mu_{0}\left(J_{y N}^{m, n}+\frac{1}{\mu_{0} \Delta} B_{z N}^{m, n}\right) \\
& \frac{i c^{2}}{\omega^{2}} \frac{k_{z}}{\Delta} E_{x N-1}^{m, n}+\frac{c^{2}}{\omega^{2}} k_{y} k_{z} E_{y N}^{m, n}+\frac{c^{2}}{\omega^{2}} \frac{E_{z N-1}^{m, n}}{\Delta^{2}}+\left[1-\frac{c^{2}}{\omega^{2}}\left(1 / \Delta^{2}+k_{y}^{2}\right)\right] E_{z N}^{m, n} \\
& =\frac{-i c^{2}}{\omega} \mu_{0}\left(J_{z N}^{m, n}-\frac{1}{\mu_{0} \Delta} B_{\nu N}^{m, n}\right)
\end{aligned}
$$

Because the impedance match is in a vacuum region, and it is assumed that no antenna source currents penetrate the plasma surface, all components at the $N$ th nodes of $\vec{J}^{m, n}$ are zero (see Fig. 13). The impedance tensor, $\overrightarrow{\overrightarrow{\mathcal{Z}}}$, described in Eqs. (A6a) and (A6b) is systematically calculated by setting $B_{y N}^{m, n}=\mu_{0}$ and $B_{z N}^{m, n}=0$ to obtain $\mathcal{Z}_{11}^{m, n}$ and $\mathcal{Z}_{21}^{m, n}$ from the $E_{y N}^{m, n}$ and $E_{z N}^{m, n}$ solutions provided by ORION-1D. The $\mathcal{Z}_{12}^{m, n}$ and $\mathcal{Z}_{22}^{m, n}$ coefficients are similarly found by setting $B_{z N}^{m, n}=\mu_{0}$ and $B_{y N}^{m, n}=0$. The impedance tensor is calculated for a desired range of $m$ and $n$ values and plasma parameters and can be used for many subsequent MAntIS runs.

At the wall opposite the plasma impedance matching location, ORION-1D makes a transition from warm to cold plasma and then applies a conducting wall boundary condition. An outgoing boundary condition is presently simulated by using an artificial absorber [5]. 


\section{REFERENCES}

[1] BATChelor D.B., CARTER M.D., GOULdiNG R.H., et al., Fusion Technology 21 (1992) 1214.

(2) GOULDING, R.H., BAITY, F.W., BATCHELOR, D.B., et al., in Radio Frequency Power in Plasmas (Proc. 9th Topical Conf. Charleston, 1991) (BATCHELOR, D.B., ed.), American Institute of Physics, New York (1992) 287 [AIP Conf. Proc. 244 (1992) 287].

[3] CARTER, M.D., BATChELOR, D.B., and JAEGER, E.F., Multiple Ar tenna Implementation System (MAntIS), ORNL/TM-12464, January 1993.

[4] BRAMBILlA, M., Nucl. Fusion 28 (1988) 549.

[5] JAEGER, E.F., BATChELOR, D.B., WEITZNER, H., Nucl. Fusion 28 (1988) 53.

[6] THEILHABER, K., JACQUINOT, J., Nucl. Fusion 24 (1984) 541.

[7] HASTE, G.R., BAITY, F.W., BARBER, G.L., et al., The folded waveguide: a highfrequency rf launcher, submitted to Fusion Eng. Des.

[8] CARTER, M.D., BAITY, F.W., BATChELOR, D.B., et al., in Radio Frequency Power in Plasmas (Proc. 9th Topical Conf. Charleston, 1991) (BATCHELOR, D.B., ed.), American Institute of Physics, New York (1992) 164 [AIP Conf. Proc. 244 (1992) 164].

[9] RYAN, P.M., BAITY, F.W., BATCHELOR, D.B., GOULDING, R.H., HOFFMAN, D.J., TOLLIVER, J.S., 18th Conference on Controlled Fusion and Plasma Physics, Vol. 15C, Part III, p. III-309 (Berlin, June 1991). 
ORNL-DWG 92M-3483 FED

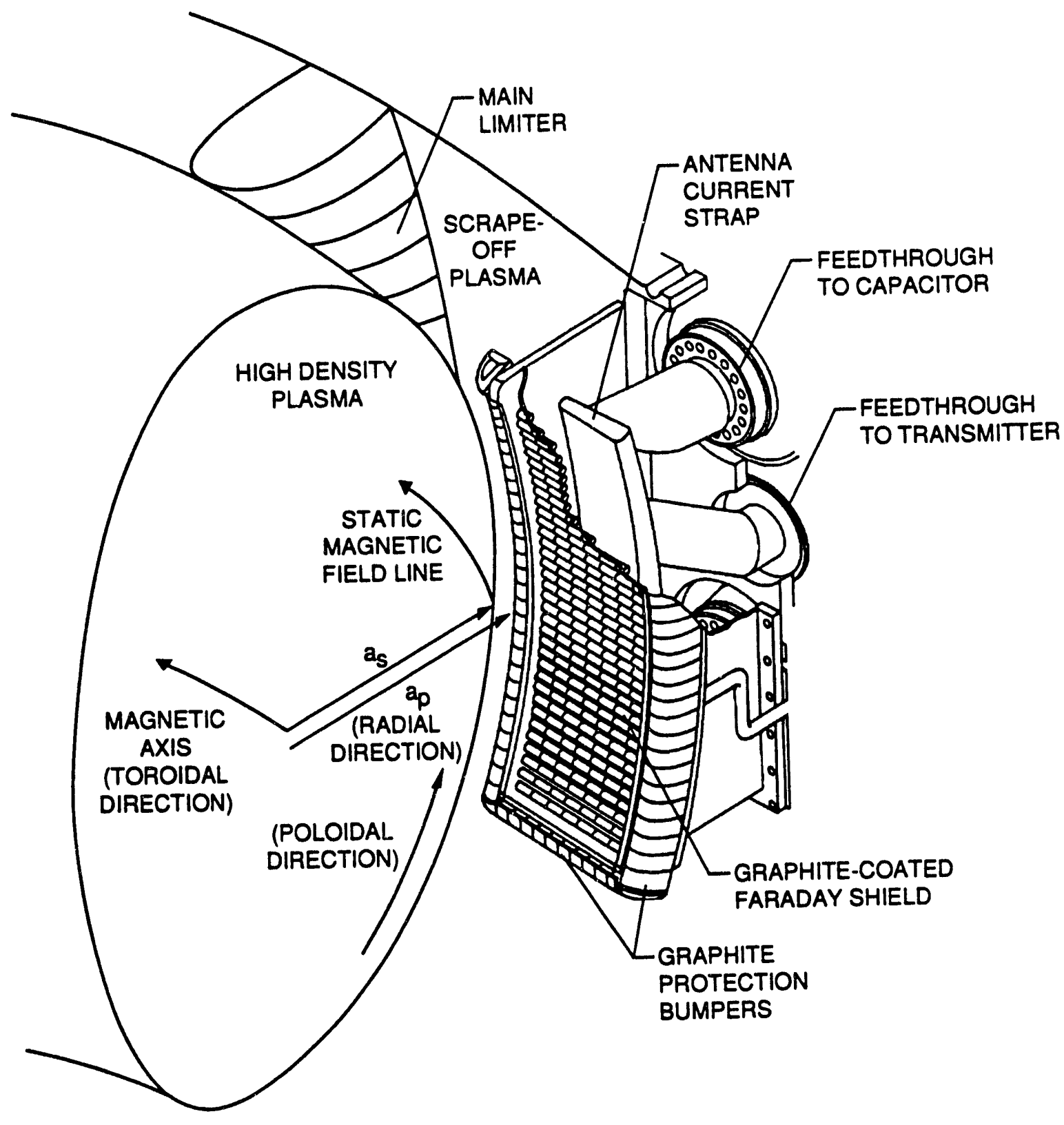

Figure 1: Schematic of a typical loop antenna design for use in a tokamak fusion experiment. 


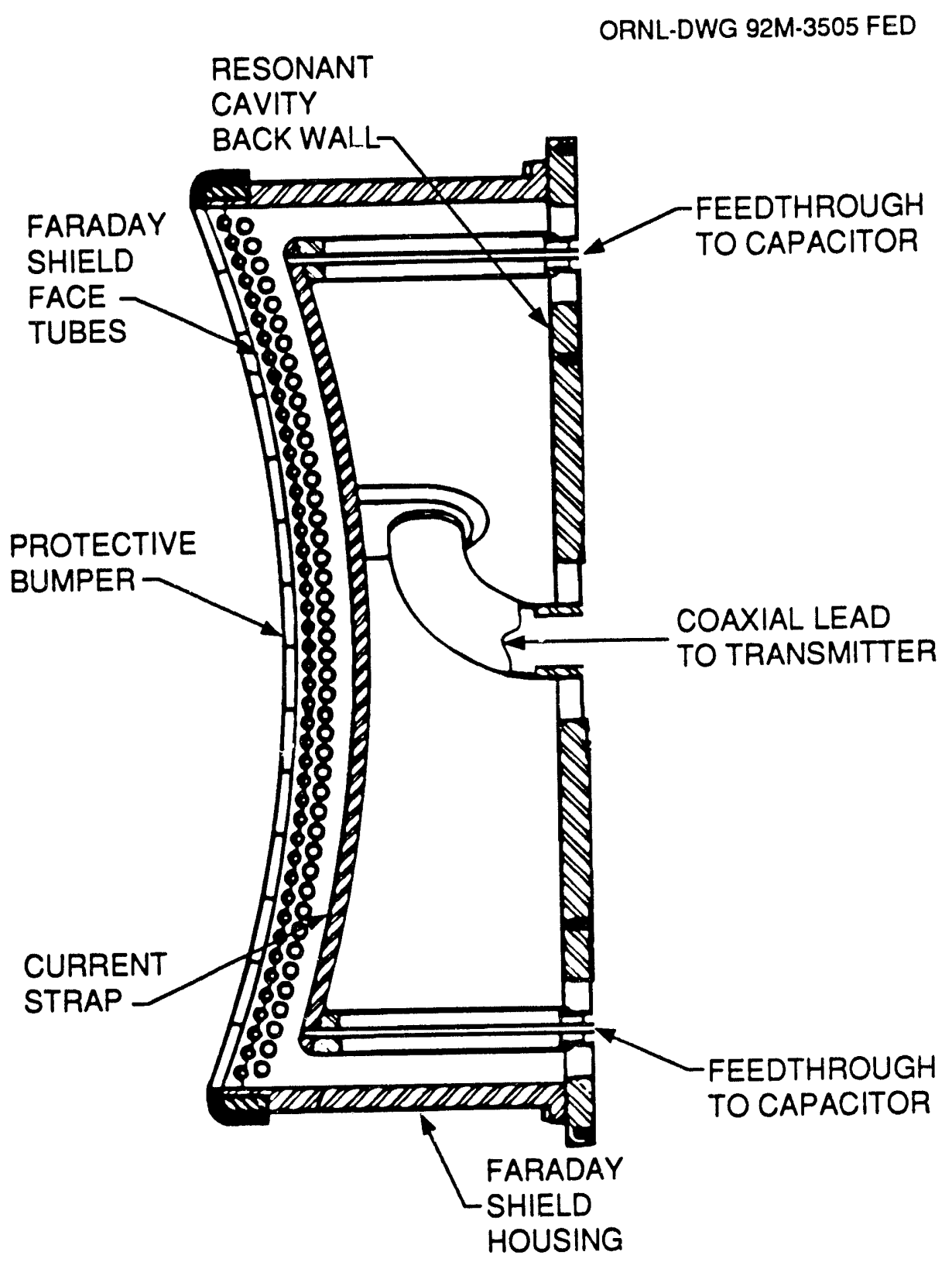

Figure 2: Parts of a loop antenna that MAntIS models. Note that the modeling of the Faraday screen is not explicit. 


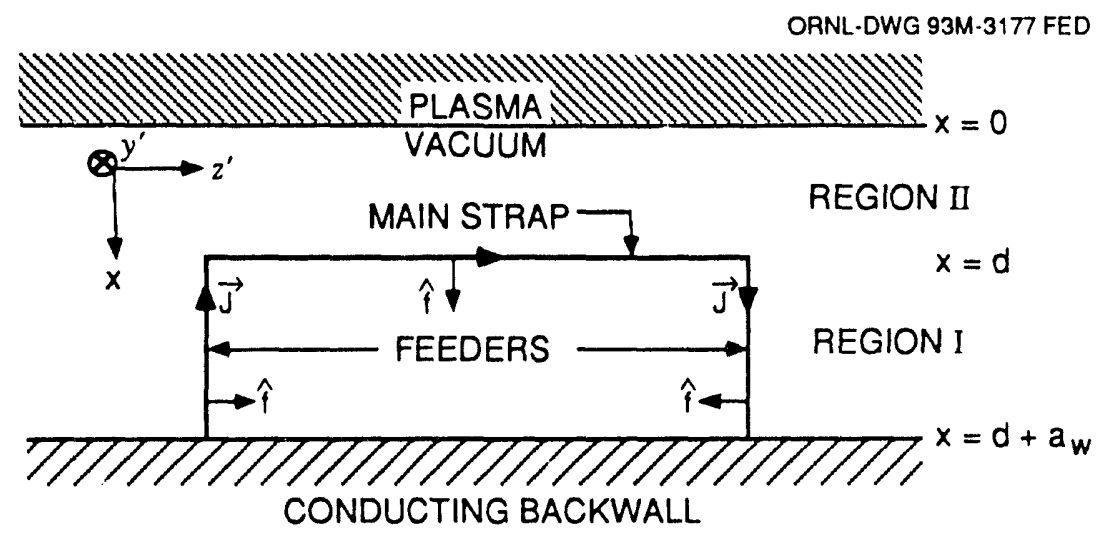

Figure 3: Cut through a MAntIS current element showing two regions for solution to Maxwell's equations. Note the direction of $\nabla f$.

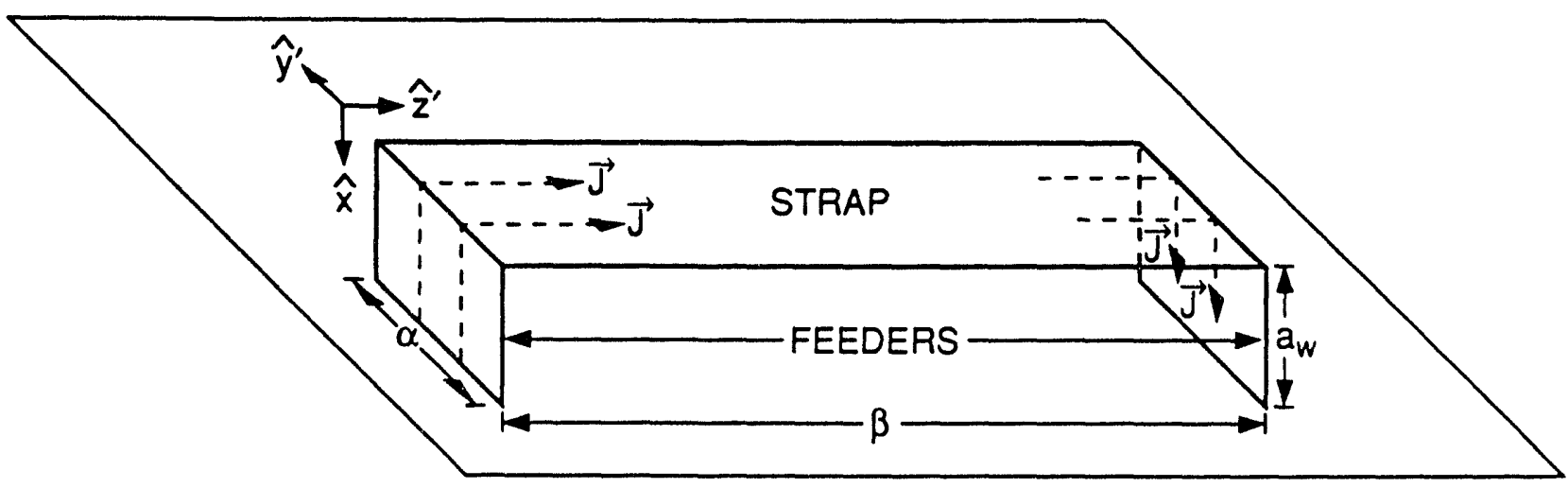

Figure 4: A perspective view of a MAntIS current element. Currents flow continuously from one feeder to the strap and from the strap back to a second feeder. 


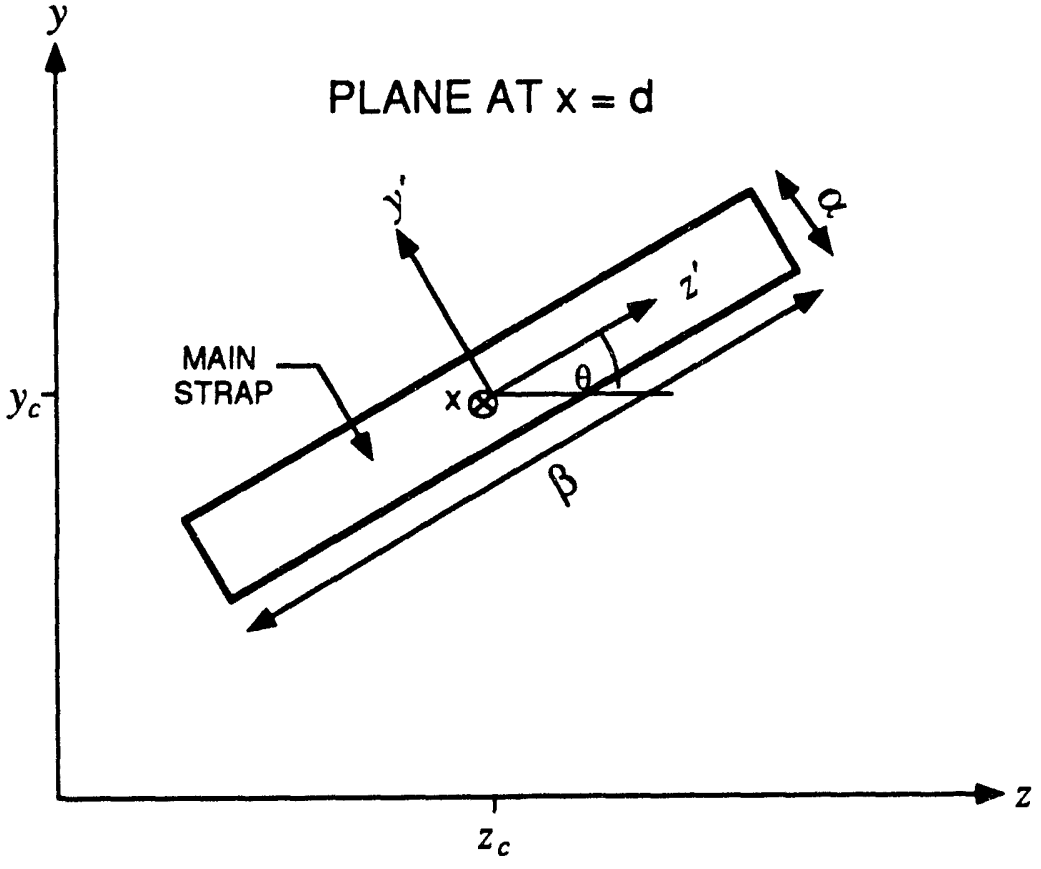

Figure 5: View of MAntIS element along the $\hat{x}$-axis. The rotation angle, $\theta$, permits arbitrary orientation relative to the toroidal direction.

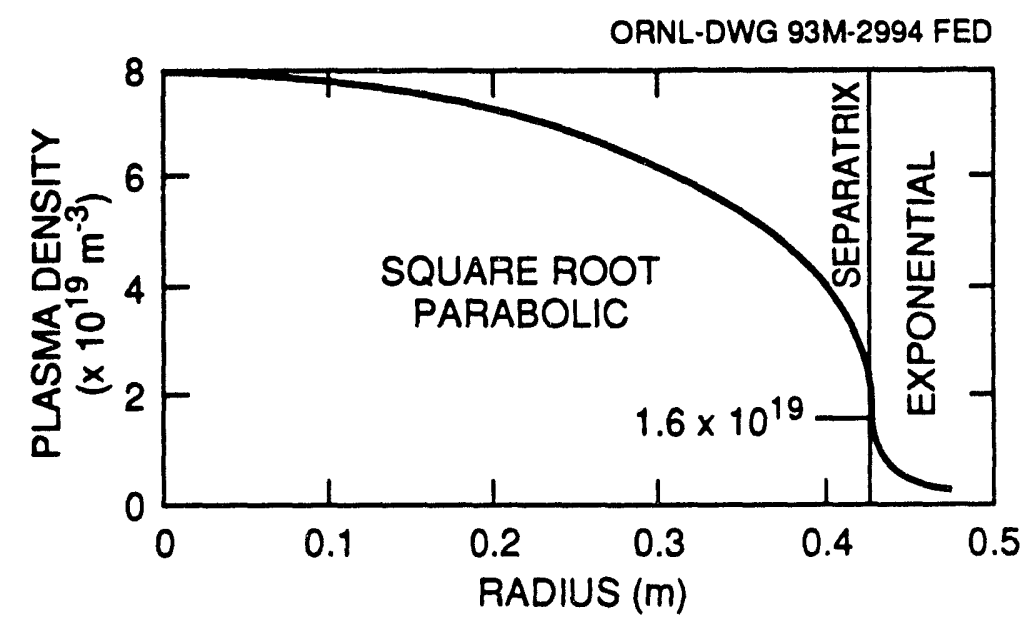

Figure 6: Plasma density profile used in all modeling. The $e$-folding length in the edge region is $0.02 \mathrm{~m}$. 


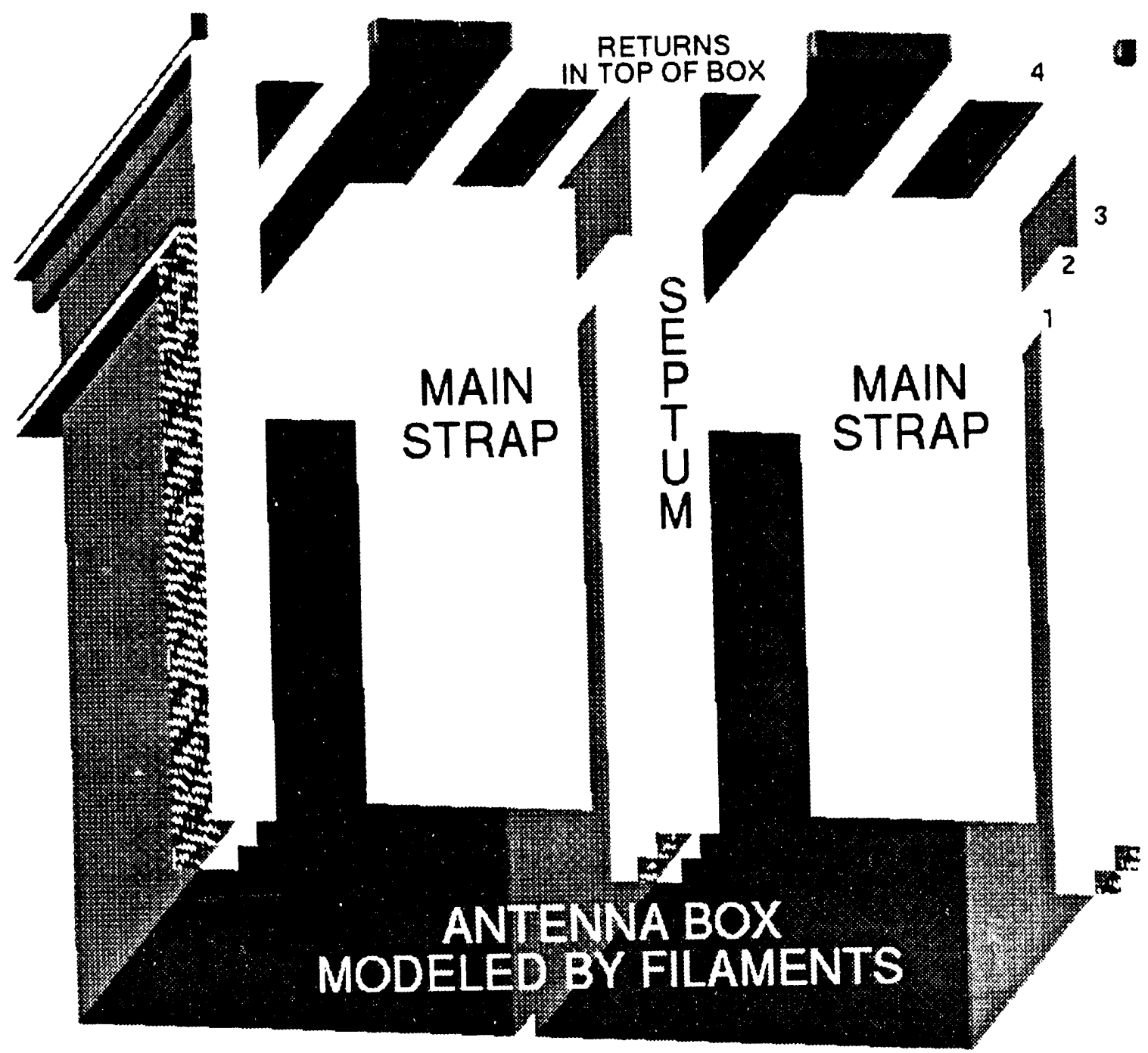

Figure 7: Location of elements used by MAntIS for 3-D modeling. Note that the mainstraps are actually represented by two filaments each, as shown in Fig. 8. Filaments 1 and 2 in the sidewall return in the sidewall. Filaments 3 and 4 cancel each other at the corner so that the return is in the top of the box. 
ORNL-DWG 93M-2995 FED

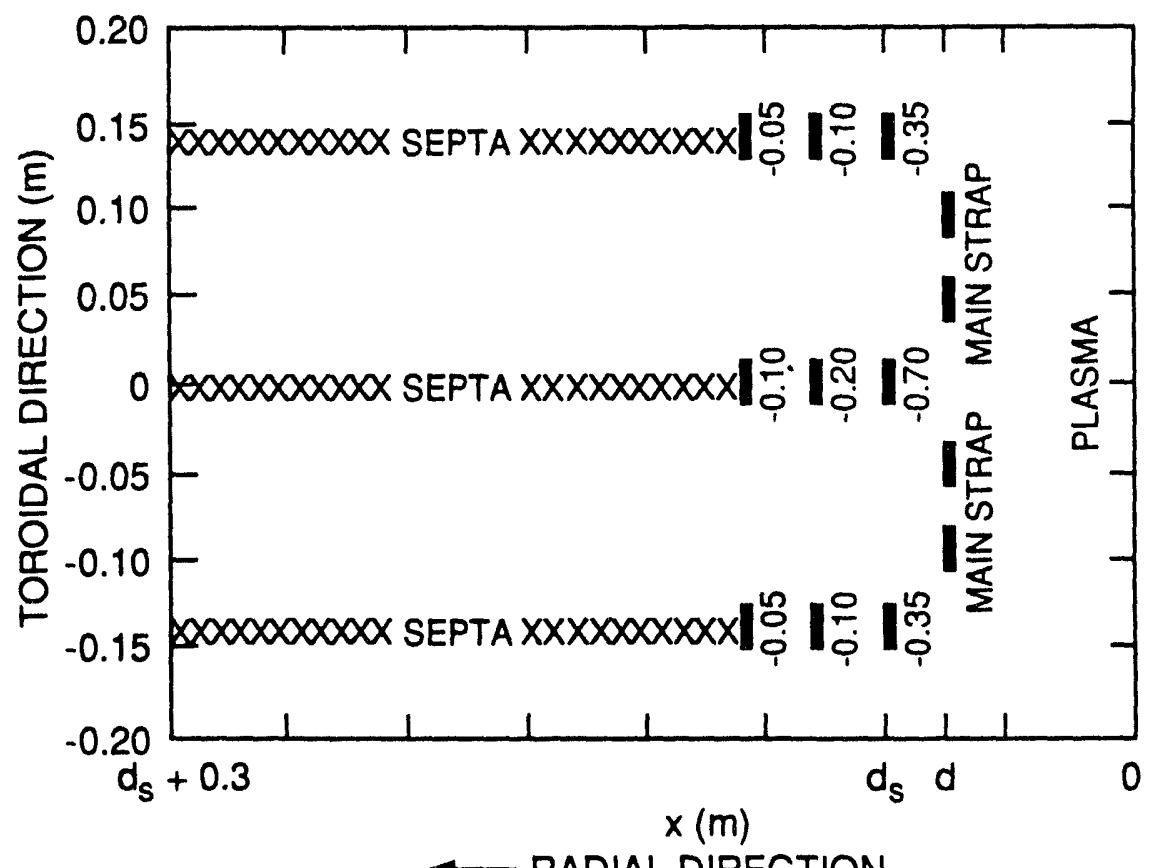

Figure 8: Schematic showing the locations of the current filaments used for the 3-D modeling in the $y=0$ plane. The numbers near the septa filaments indicate the amplitude of the current in that filament. 


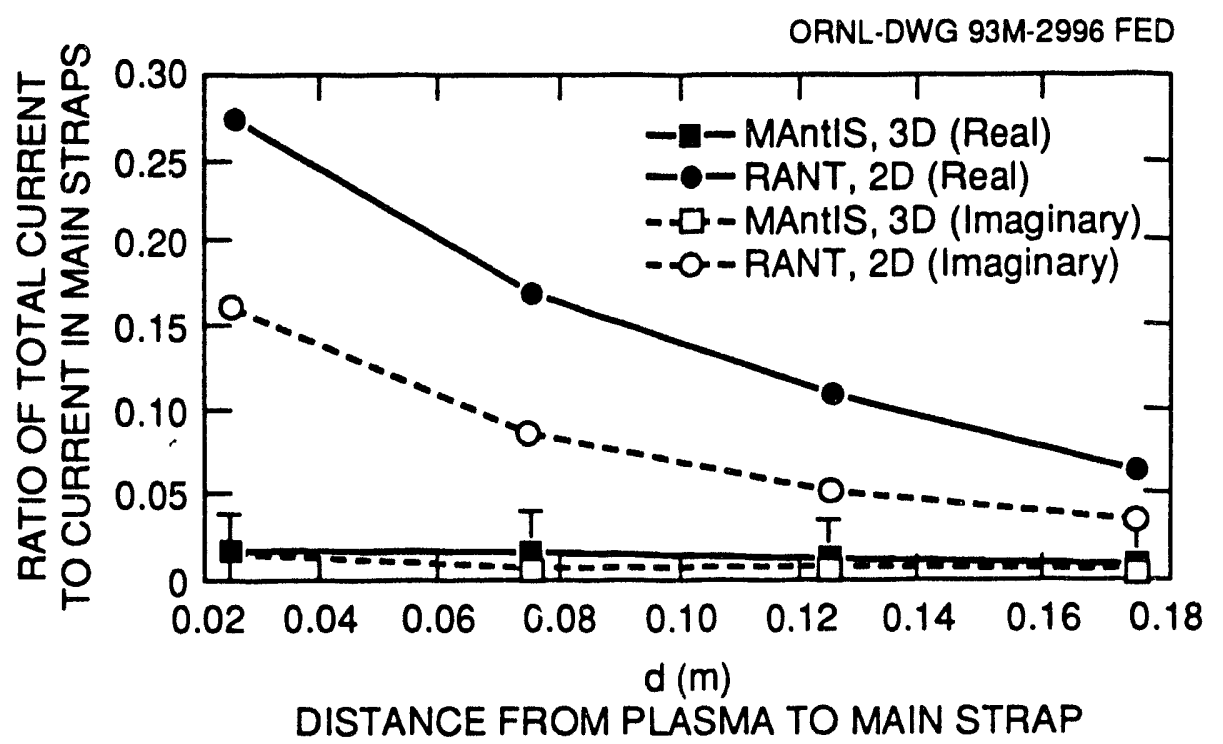

Figure 9: Feeders to the mainstraps and returns in the septum and sidewalls provide the low inductance path for return currents. This path is present only in 3-D models and prevents current from being "robbed" by the plasma, as happens in the 2-D limit. Errors in the MAntIS calculations occur because of the finite number of poloidal modes used to calculate currents in the conducting backwall.

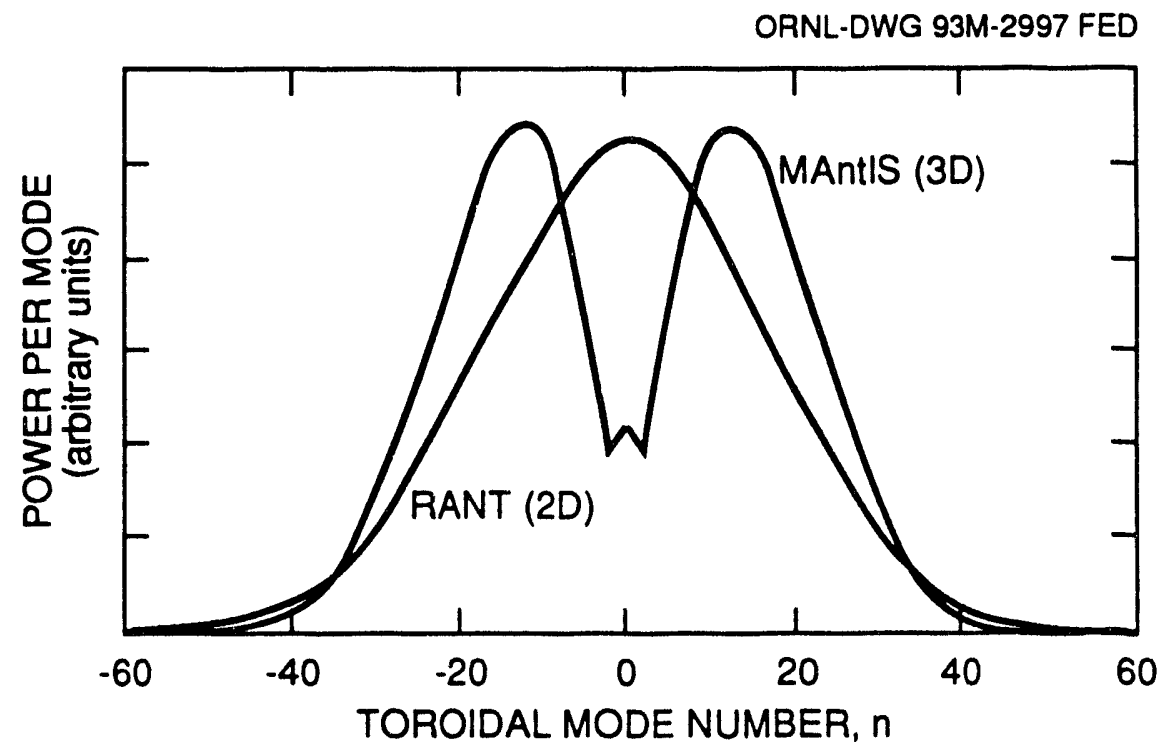

Figure 10: Average over the poloidal modes for the launched power spectrum in the 3-D model shows a depression near $n=0$ caused by return currents in the septum and sidewalls. The 2-D calculation does not show this depression because some of the return current is "robbed" by the plasma. 


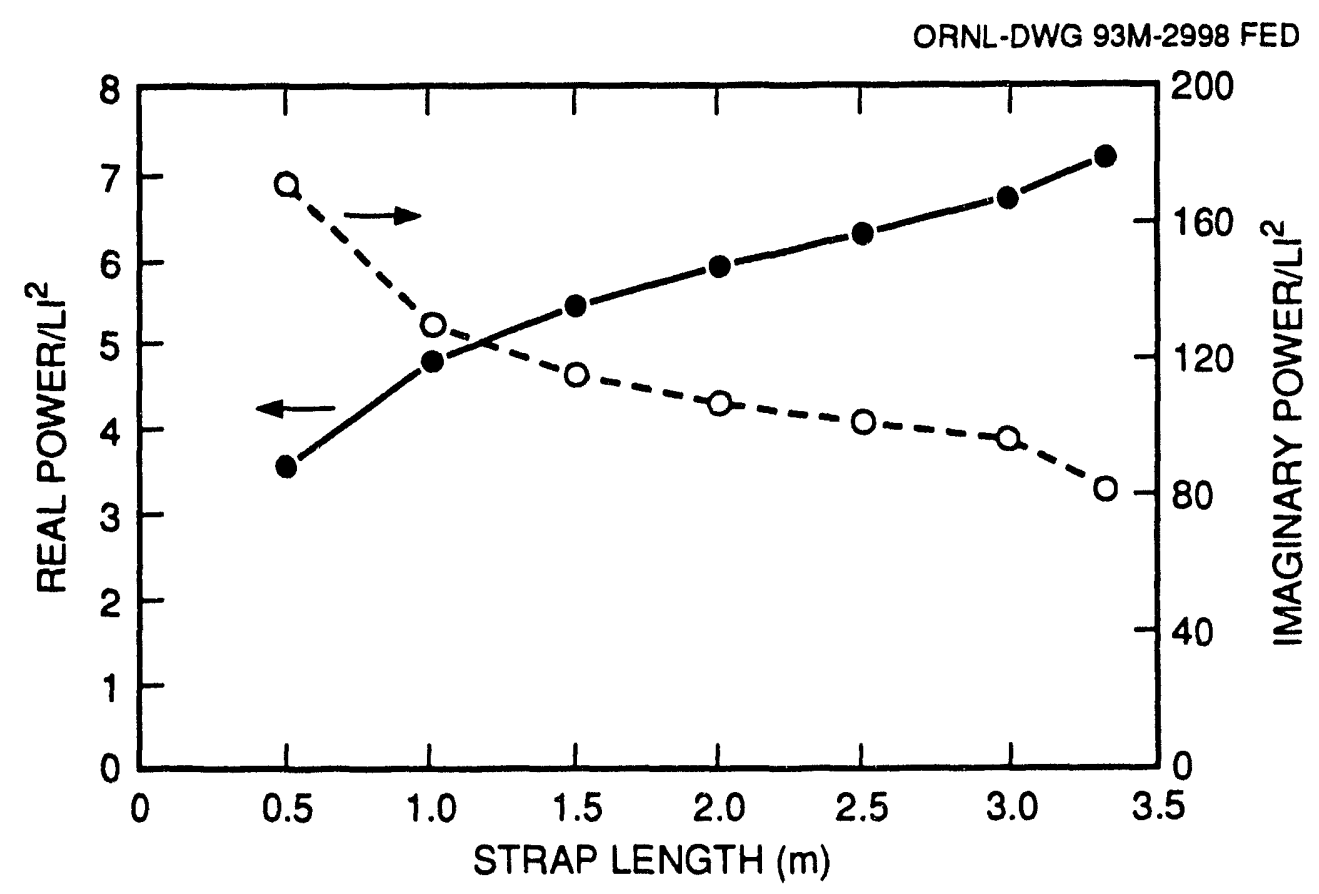

Figure 11: Poloidal modes excited by finite-length antennas do not propagate as well as the $m=0$ poloidal mode ( $2-\mathrm{D}$ limit), resulting in a reduction in loading for plasmas with density near the fast wave cutoff. 


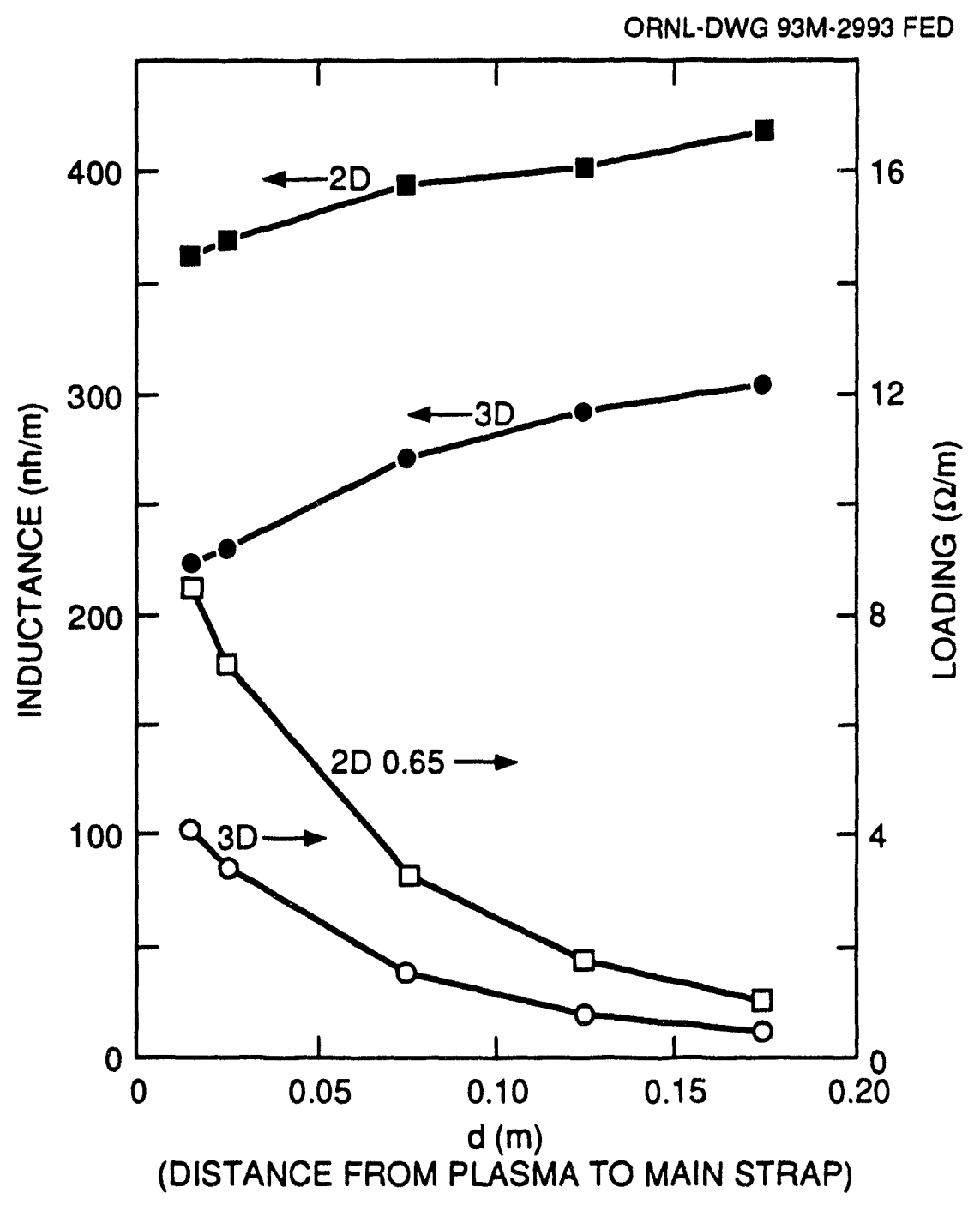

Figure 12: For the plasma parameters considered here, both the inductance and the real loading are reduced in the 3-D model. For higher plasma edge density, the loading is not so strongly affected. 


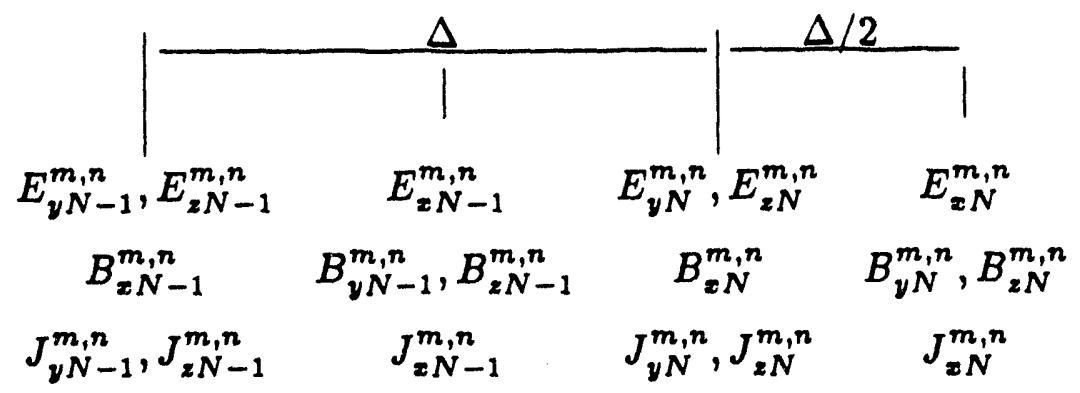

Figure 13: Grid used for finite differencing in the ORION. $1 \mathrm{D}$ code to calculate the impedance match between the plasma and vacuum regions. 
ORNL/TM-12464

Dist. Category UC-427

\section{INTERNAL DISTRIBUTION}

1. Director, ORNL Fusion Energy Division

2. C. C. Baker

3. L. A. Berry

4. B. A. Carreras

5. H. E. Clark

6. R. I. Colchin

7. R. A. Dory

8. J. L. Dunlap

9. F. E. Gethers

10. T. E. Shannon

11. W. L. Stirling

12-13. Laboratory Records Department

14. Laboratory Records, ORNL-RC

15-16. Central Research Library

17. Document Reference Section

18. Fusion Energy Division Library

19-20. ET/FE Division Publications Office

21. ORNL Patent Office
22-26. D. B. Batchelor

27-31. M. D. Carter

32. F. W. Baity

33. T. S. Bigelow

34. R. C. Goldfinger

35. R. H. Goulding

36. S. P. Hirshman

37. D. J. Hoffman

38. W. A. Houlberg

39. E. F. Jaeger

40. S. L. Milora

41. D. A. Rasmussen

42. P. M. Ryan

43. D. C. Stallings

44. D. W. Swain

45. J. H. Whealton

46. J. J. Yugo

\section{EXTERNAL DISTRIBUTION}

47. Office of the Assistant Manager for Energy Research and Development, Department of Energy, Oak Ridge Operations, P.O. Box 2000, Oak Ridge, TN 37831

48. N. A. Davies, Director, Office of Fusion Energy, Office of Energy Research, ER-50 Germantown, U.S. Department of Energy, Washington, DC 20545

49. M. Roberts, International Programs, Office of Fusion Energy, Office of Energy Research, ER-52 Germantown, U.S. Department of Energy, Washington, DC 20545

50. D. E. Baldwin, Lawrence Livermore National Laboratory, P.O. Box 5511, Livermore, CA 94550

51. R. W. Conn, Mechanical, Aerospace, and Nuclear Engineering Department, 6291 Boelter Hall, University of California, Los Angeles, CA 90024-1597

52. P. C. Liewer, MS 138-208, Jet Propulsion Laboratory, 4800 Oak Grove Drive, Pasadena, CA 91109

53. D. Sigmar, Plasma Fusion Center, Massachusetts Institute of Technology, 167 Albany St., NW 16-288, Cambridge, MA 02139

54. K. I. Thomassen, L-637, Lawrence Livermore National Laboratory, P.O. Box 5511, Livermore, CA 94550

55. J. D. Callen, Department of Nuclear Engineering, University of Wisconsin, Madison, WI 53706-1687

56. S. O. Dean, Fusion Power Associates, Inc., 2 Professional Drive, Suite 248, Gaithersburg, MD 20879

57. H. K. Forsen, Bechtel Group, Inc., Research Engineering, P.O. Box 3965, San Francisco, CA 94119 
58. R. W. Gould, Department of Applied Physics, California Institute of Technology, Pasadena, CA 91125

59. R. A. Gross, Plasma Research Laboratory, Columbia University, New York, NY 10027

60. R. J. Hawryluk, Plasma Physics Laboratory, Princeton University, P.O. Box 451, Princeton, NJ 08543

61. D. M. Meade, Plasma Physics Laboratory, Princeton University, P.O. Box 451, Princeton, NJ 08543

62. W. M. Stacey, School of Nuclear Engineering and Health Physics, Georgia Institute of Technology, Atlanta, GA 30332

63. D. Steiner, Nuclear Engineering Department, NES Building, Tibbetts Avenue, Rensselaer Polytechnic Institute, Troy, NY 12181

64. R. Varma, Physical Research Laboratory, Navrangpura, Ahmedabad 380009, India

65. Bibliothek, Max-Planck Institut für Plasmaphysik, Boltzmannstrasse 2, D-8046 Garching, Federal Republic of Germany

66. Bibliothek, Institut für Plasmaphysik, KFA Jülich GmbH, Postfach 1913, D-5170 Jülich, Federal Republic of Germany

67. Bibliothek, KfK Karlsruhe GmbH, Postfach 3640, D-7500 Karlsruhe 1, Federal Republic of Germany

68. Bibliotheque, Centre de Recherches en Physique des Plasmas, Ecole Polytechnique Fédérale de Lausanne, 21 Avenue des Bains, CH-1007 Lausanne, Switzerland

69. R. Aymar, CEN/Cadarache, Departement de Recherches sur la Fusion Contrôlée, F-13108 Saint-Paul-lez-Durance Cedex, France

70. Bibliothèque, CEN/Cadarache, F-13108 Saint-Paul-lez-Durance Cedex, France

71. Library, JET Joint Undertaking, Abingdon, Oxfordshire OX14 3EA, England

72. Library, FOM-Instituut voor Plasmafysica, Rijnhuizen, Edisonbaan 14, 3439 MN Nieuwegein, The Netherlands

73. Library, National Institute for Fusion Science, Chikusa-ku, Nagoya 464-01, Japan

74. Library, International Centre for Theoretical Physics, P.O. Box 586, I-34100 Trieste, Italy

75. Library, Centro Richerche Energia Frascati, C.P. 65, I-00044 Frascati (Roma), Italy

76. Library, Plasma Physics Laboratory, Kyoto University, Gokasho, Uji, Kyoto 611, Japan

77. Plasma Research Laboratory, Australian National University, P.O. Box 4, Canberra, A.C.T. 2601, Australia

78. Library, Japan Atomic Energy Research Institute, Naka Fusion Research Establishment, 801-1 Mukoyama, Naka-machi, Naka-gun, Ibaraki-ken, Japan

79. G. A. Eliseev, I. V. Kurchatov Institute of Atomic Energy, P.O. Box 3402, 123182 Moscow, Russia

80. V. A. Glukhikh, Scientific-Research Institute of Electro-Physical Apparatus, 188631 St. Petersburg, Russia

81. I. Shpigel, Institute of General Physics, U.S.S.R. Academy of Sciences, Ulitsa Vavilova 38, Moscow, Russia

82. D. D. Ryutov, Institute of Nuclear Physics, Siberian Academy of Sciences, Sovetskaya St. 5, 630090 Novosibirsk, Russia

83. O. Pavlichenko, Kharkov Physical-Technical Institute, Academical St. 1, 310108 Kharkov, Ukraine

84. Deputy Director, Southwestern Institute of Physics, P.O. Box 15, Leshan, Sichuan, China (PRC)

85. Director, The Institute of Plasma Physics, P.O. Box 1126, Hefei, Anhui, China (PRC)

86. R. H. McKnight, Experimental Plasma Research Branch, Division of Development and Technology, Office of Fusion Energy, Office of Energy Research, ER-542 Germantown, U.S. Department of Energy, Washington, DC 20545

87. D. Crandall, Director, Division of Applied Plasma Physics, Office of Fusion Energy, Office of Energy Research, ER-54 Germantown, U.S. Department of Energy, Washington, DC 20545 
88. E. Oktay, Division of Confinement Systems, Office of Fusion Energy, Office of Energy Research, ER-55 Germantown, U.S. Department of Energy, Washington, DC 20545

89. W. Sadowski, Fusion Theory and Computer Services Branch, Division of Applied Plasma Physics, Office of F usion Energy, Office of Energy Research, ER-541 Germantown, U.S. Department of Energy, Washington, DC 20545

90. R. E. Mickens, Atlanta University, Department of Physics, Atlanta, GA 30314

91. M. N. Rosenbluth, University of California at San Diego, La Jolla, CA 92037

92. D. Schnack, SAIC, 10260 Campus Point Drive, San Diego, CA 92121

93. Duk-In Choi, Department of Physics, Korea Advanced Institute of Science and Technology, P.O. Box 150, Chong Ryang-Ri, Seoul, Korea

94. Library of Physics Department, University of Ioannina, Ioannina, Greece

95. C. De Palo, Library, Associazione EURATOM-ENEA sulla Fusione, CP 65, I-00044 Frascati (Roma), Italy

96. Laboratorio Associado de Plasma, Instituto Nacional de Pesquisas Espaciais, Caixa Postal 515, 122201, Sao Jose dos Campos, SP, Brazil

97. Theory Department Read File, c/o D. W. Ross, University of Texas, Institute for Fusion Studies, Austin, TX 78712

98. Theory Department Read File, clo R. Parker, Director, Plasma Fusion Center, NW 16-202, Massachusetts Institute of Technology, Cambridge, MA 02139

99. Theory Department Read File, c/o R. White, Plasma Physics Laboratory, Princeton University, P.O. Box 451, Princeton, NJ 08543

100. Theory Department Read File, c/o L. Kovrizhnykh, Lebedev Institute of Physics, Academy of Sciences, 53 Leninsky Prospect, 117924 Moscow, Russia

101. Theory Department Read File, c/o B. B. Kadomtsev, I. V. Kurchatov Institute of Atomic Energy, P.O. Box 3402, 123182 Moscow, Russia

102. Theory Department Read File, c/o T. Kamimura, National Institute for Fusion Studies, Nagoya 464, Japan

103. Theory Department Read File, c/o E. Maschke, Departemente de Recherches sur la Fusion Controlee, CEN/Cadarache, F-13108 Saint-Paul-lez Durance, France

104. Theory Departınent Read File, c/o D. Düchs, JET Joint Undertaking, Abingdon, Oxfordshire OX14 3EA, United Kingdom

105. Theory Department Read File, c/o R. Briscoe, Culham Laboratory, Abingdon, Oxfordshire OX14 3DB, United Kingdom

106. Theory Department Read File, c/o D. Biskamp, Max-Planck-Institut fur Plasmaphysik, Boltzmannstrasse 2, D-8046 Garching, Federal Republic of Germany

107. Theory Department Read File, c/o T. Takeda, Japan Atomic Energy Research Institute, Tokai Fusion Research Establishment, Tokai-mura, Naka-gun, Ibaraki-ken, Japan

108. Theory Department Read File, c/o J. Greene, General Atomics, P.O. Box 85608, San Diego, CA 92186

109. Theory Department Read File, c/o R. Cohen, Lawrence Livermore National Laboratory, P.O. Box 5511, Livermore, CA 94550

110. Theory Department Read File, c/o R. Gerwin, CTR Division, Los Alamos National Laboratory, P.O. Box 1663, Los Alamos, NM 87545

111. R. Aamodt, Lodestar Research Corporation, 2400 Central Avenue, Boulder, CO 80301

112. K. Appert, Centre de Recherches en Physique des Plasmas, Ecole Polytechnique Fédérale de Lausanne, 21 Avenue des Bains, CH-1007 Lausanne, Switzerland

113. M. Ballico, Max-Planck Institut für Plasmaphysik, Boltzmannstrasse 2. D-8046 Garching, Federal Republic of Germany

114. E. Barbato, Associazione EURATOM-ENEA sulla Fusione, Centro Richerche Energia Frascati, C.P. 65, I-00044 Frascati (Roma), Italy

115 A. Bécoulet, Association EURATOM-CEA sur la Fusion Controlée, Centre d'Etudes Nucléaires de Cadarache, B. P. No. 1, F-13108 Saint Paul lez Durances, France 
116. S. Bernabei, Plasma Physics Laboratory, Princeton University, P.O. Box 451, Princeton, NJ 08543

117. A. Bers, Plasma Fusion Center, Massachusetts Institute of Technology, 38-260, Cambridge, MA 02139

118. V. Bhatnagar, JET Joint Undertaking, Abingdon, Oxfordshire OX14 3EA, England

119. P. T. Bonoli, Plasma Fusion Center, Massachusetts Institute of Technology, 175 Albany St., NW17-115, Cambridge, MA 02139

120. M. Brambilla, Max-Planck Institut für Plasmaphysik, Boltzmannstrasse 2, D-8046 Garching, Federal Republic of Germany

121. Dr. Vincent Chan, General Atomics, P. O. Box 85608, San Diego, CA 92186

122. D. Ehst, Building 205, Argonne National Laboratory, 9700 S. Cass Avenue, Argonne, IL 60538

123. D. Faulconer, Laboratoire de Physique des Plasmas, EURATOM-Etat Belge, Ecole Royale Militaire, Avenue de la Renaissance 30, 1040 Brussels, Belgium

124. D. J. Gambier, JET Joint Undertaking, Abingdon, Oxfordshire OX 14 3EA, England

125. S. N. Golovato, Plasma Fusion Center, Massachusetts Institute of Technology, 175 Albany Street, NW17-113, Cambridge, MA 02139

126. W. Grossman, SAIC, 1710 Goodridge Drive, McLean, VA 22102

127. W. Ho, SAIC, 10260 Campus Point Drive, San Diego, CA 92121

128. J. Hosea, Plasma Physics Laboratory, Princeton University, P.O. Box 451, Princeton, NJ 08543

129. Kwok Ko, Stanford Linear Accelerator Center, P.O. Box 4349, Stanford, CA 94309

130. C. C. Petty, General Atomics, P.O. Box 85608, San Diego, CA 92186

131. C. K. Phillips, Plasma Physics Laboratory, Princeton University, P.O. Box 451, Princeton, NJ 08543

132. M. Porkolab, Plasma Fusion Center, Massachusetts Institute of Technology, 175 Albany Street, NW17-119, Cambridge, MA 02139

133. R. Prater, General Atomics, P.O. Box 85608, San Diego, CA 92186

134. J. Scharer, Electrical and Computer Engineering Department, 1500 Johnson Drive, University of Wisconsin, Madison, WI 53706

135. D. C. Stevens, Courant Institute for Mathematical Sciences, New York University, 251 Mercer Street, New York, NY 10001

136. P. E. Vandenplas, Laboratoire de Physique des Plasmas, EURATOM-Etat Belge, Ecole Royale Militaire, Avenue de la Renaissance 30, 1040 Brussels, Belgium

137. H. Weitzner, Courant Institute for Mathematical Sciences, New York University, 251 Mercer Street, New York, NY 10001

138. J. R. Wilson, Plasma Physics Laboratory, Princeton University, P.O. Box 451, Princeton, NJ 08543

139-186. Given distribution according to OSTI-4500, Magnetic Fusion Energy (Category Distribution UC-427, Theoretical Plasma Physics) 

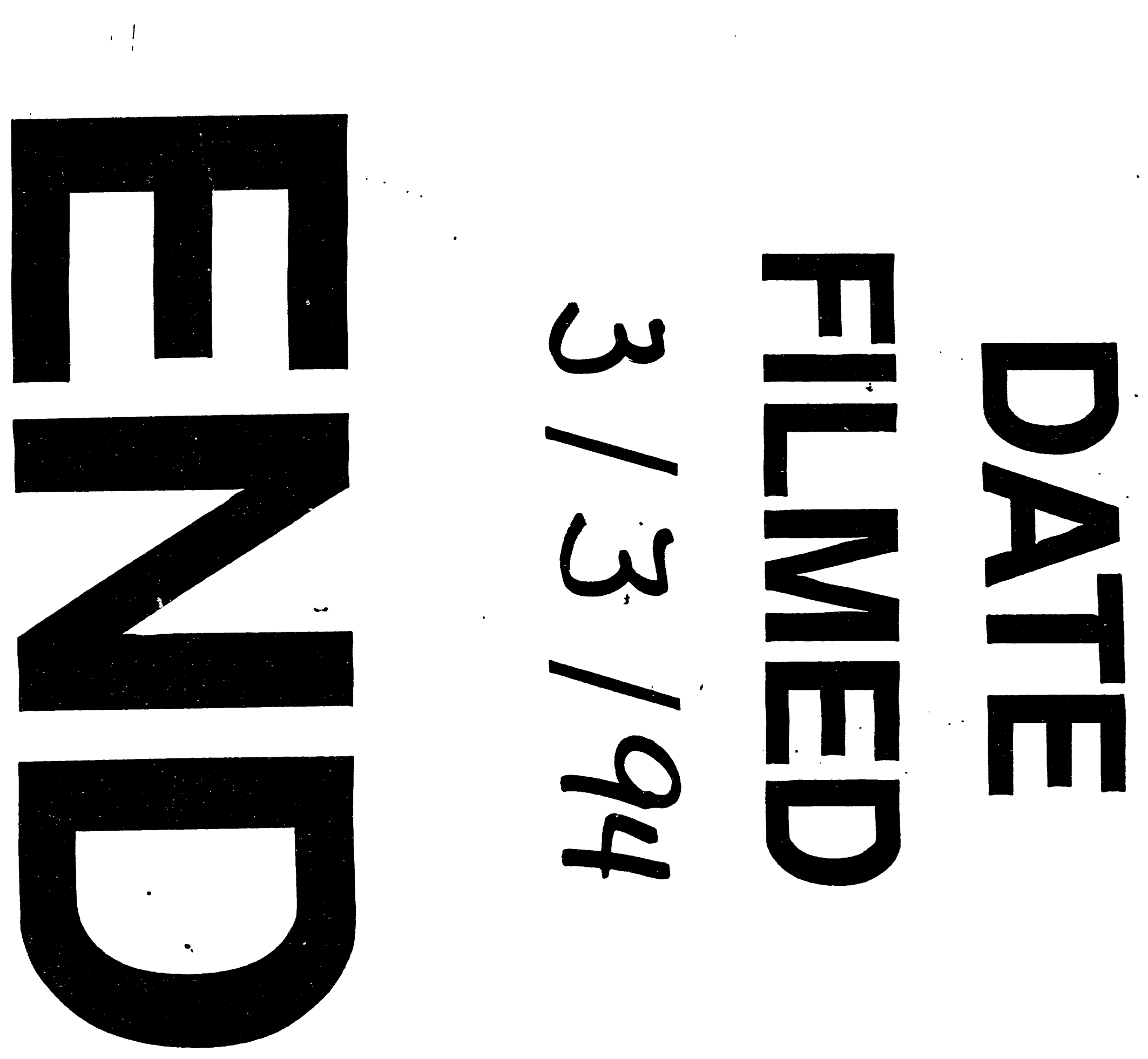